
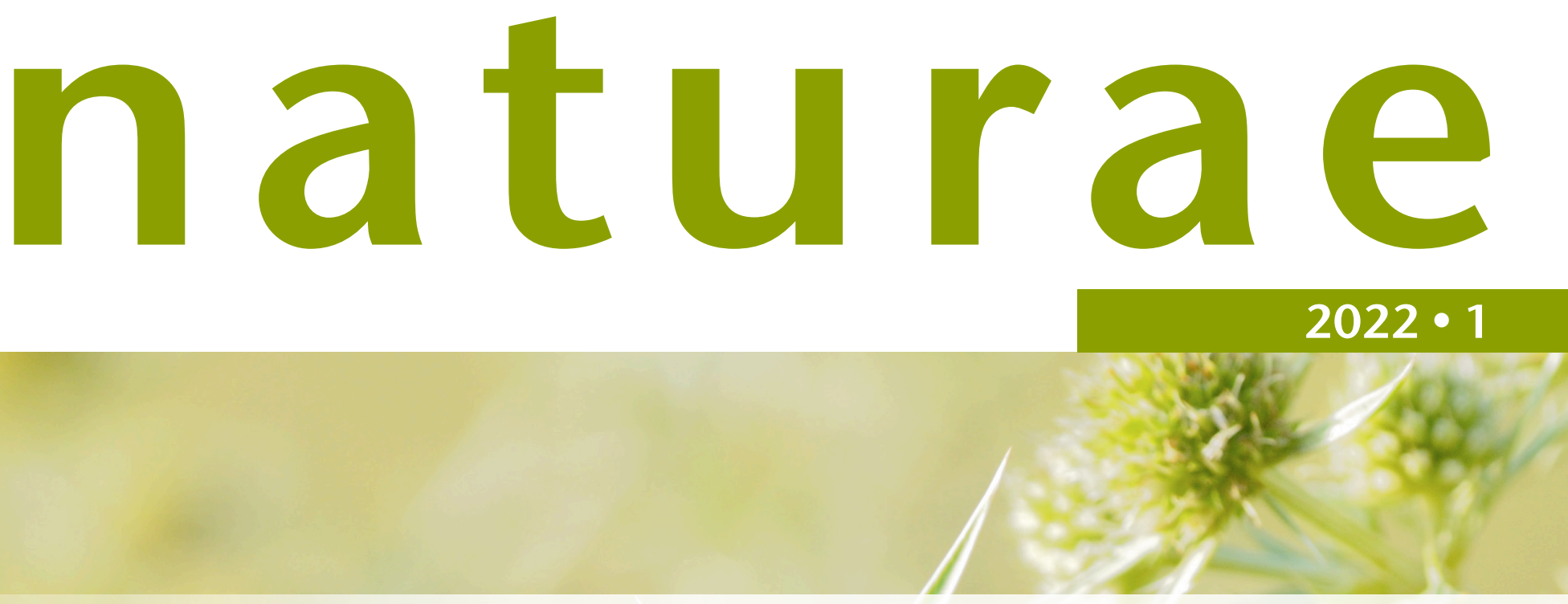

Écologie et conservation du Mercure Arethusana arethusa (Denis \& Schiffermüller, 1775) (Lépidoptère: Satyrinae) en Hauts-de-France

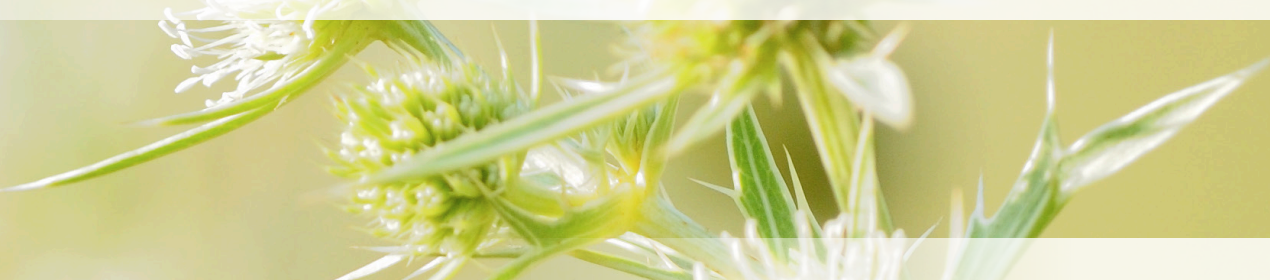

Guillaume MEIRE \& Marc BRUNEAU

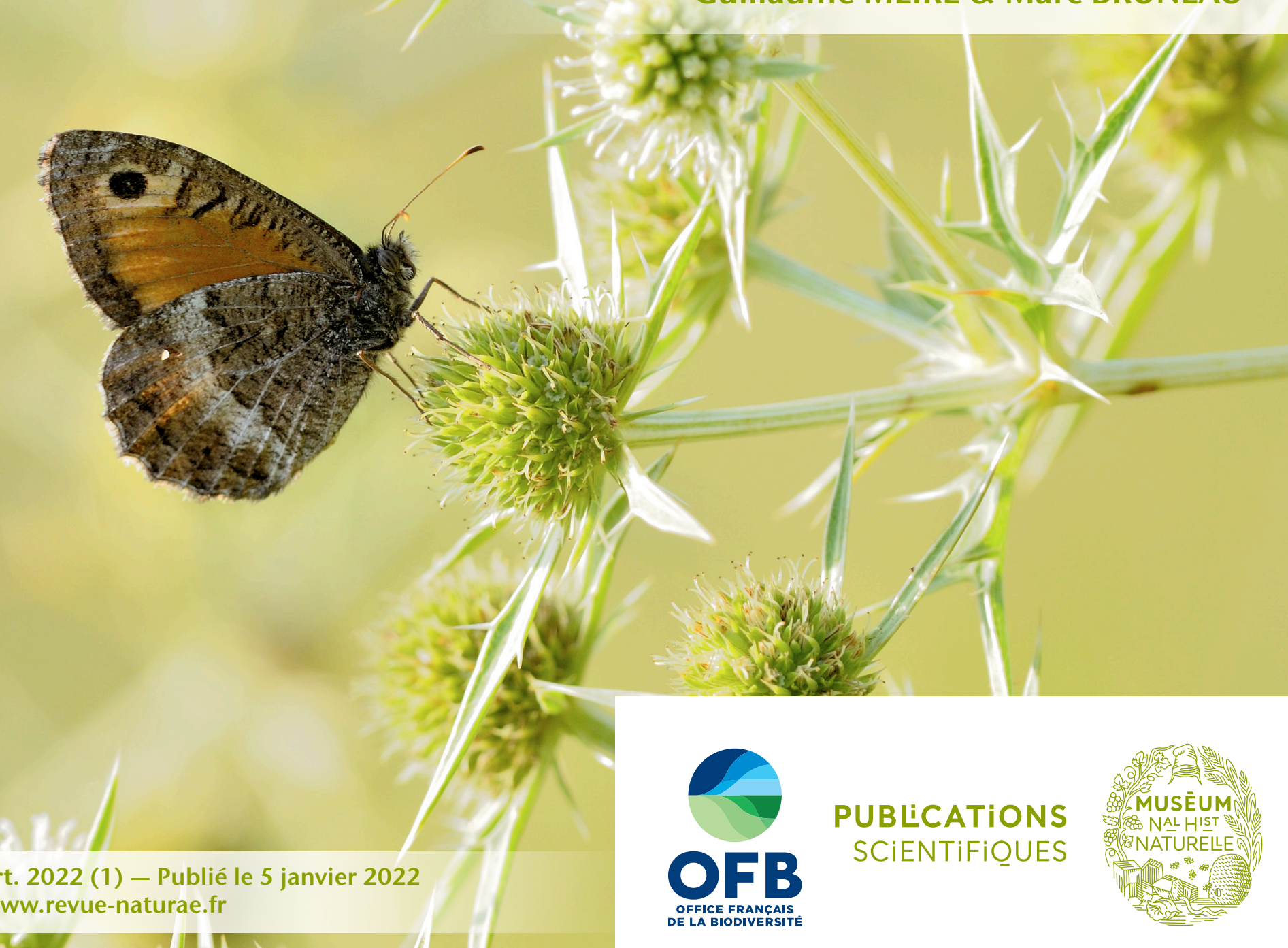


DiRECTEUR DE LA PUBLICATION / PUBLICATION DIRECTOR: Bruno David,

Président du Muséum national d'Histoire naturelle

RÉDACTEUR EN CHEF / EDITOR-IN-CHIEF: Jean-Philippe Siblet

ASSISTANTE DE RÉDACTION / ASSISTANT EDITOR: Sarah Figuet (naturae@mnhn.fr)

Mise EN PAGE / PAGE LAYOUT: Sarah Figuet

COMITÉ SCIENTIFIQUE / SCIENTIFIC BOARD:

Luc Abbadie (UPMC, Paris)

Luc Barbier (Parc naturel régional des caps et marais d'Opale, Colembert)

Aurélien Besnard (CEFE, Montpellier)

Vincent Boullet (Expert indépendant flore/végétation, Frugières-le-Pin)

Hervé Brustel (École d'ingénieurs de Purpan, Toulouse)

Patrick De Wever (MNHN, Paris)

Thierry Dutoit (UMR CNRS IMBE, Avignon)

Éric Feunteun (MNHN, Dinard)

Romain Garrouste (MNHN, Paris)

Grégoire Gautier (DRAAF Occitanie, Toulouse)

Olivier Gilg (Réserves naturelles de France, Dijon)

Frédéric Gosselin (Irstea, Nogent-sur-Vernisson)

Patrick Haffner (PatriNat, Paris)

Frédéric Hendoux (MNHN, Paris)

Xavier Houard (OPIE, Guyancourt)

Isabelle Leviol (MNHN, Concarneau)

Francis Meunier (Conservatoire d'espaces naturels - Hauts-de-France, Amiens)

Serge Muller (MNHN, Paris)

Francis Olivereau (DREAL Centre, Orléans)

Laurent Poncet (PatriNat, Paris)

Nicolas Poulet (OFB, Vincennes)

Jean-Philippe Siblet (PatriNat, Paris)

Laurent Tillon (ONF, Paris)

Julien Touroult (PatriNat, Paris)

COUVERTURE / COVER:

Le Mercure Arethusana arethusa (Denis \& Schiffermüller, 1775) posé sur un Panicaut champêtre. Crédit photo: Patrick Carliez.

Naturae est une revue en flux continu publiée par les Publications scientifiques du Muséum, Paris

Naturae is a fast track journal published by the Museum Science Press, Paris

Les Publications scientifiques du Muséum publient aussi / The Museum Science Press also publish:

Adansonia, Zoosystema, Anthropozoologica, European Journal of Taxonomy, Geodiversitas, Cryptogamie sous-sections Algologie, Bryologie, Mycologie, Comptes Rendus Palevol.

Diffusion - Publications scientifiques Muséum national d'Histoire naturelle

CP 41 - 57 rue Cuvier F-75231 Paris cedex 05 (France)

Tél. : 33 (0)1 40794805 / Fax: 33 (0)1 40793840

diff.pub@mnhn.fr / https://sciencepress.mnhn.fr

(c) Publications scientifiques du Muséum national d'Histoire naturelle, Paris, 2022

ISSN (électronique / electronic): 1638-9387 


\title{
Écologie et conservation du Mercure Arethusana arethusa (Denis \& Schiffermüller, 1775) (Lépidoptère: Satyrinae) en Hauts-de-France
}

\author{
Guillaume MEIRE \\ Conservatoire d'Espaces naturels des Hauts-de-France, \\ 1 place Gingko - village Oasis, F-80044 Amiens Cedex 1 (France) \\ g.meire@cen-hautsdefrance.org \\ Marc BRUNEAU \\ 91 rue des Marchis, F-36500 Buzancais (France) \\ bruneau-marc@outlook.fr
}

Soumis le 13 novembre 2020 | Accepté le 8 mars 2021 | Publié le 5 janvier 2022

MOTS CLÉS

Capture-marquage

-recapture,

habitat,

papillon de jour

pelouse calcicole, gestion conservatoire.
Meire G. \& Bruneau M. 2022. - Écologie et conservation du Mercure Arethusana arethusa (Denis \& Schiffermüller, 1775) (Lépidoptère: Satyrinae) en Hauts-de-France. Naturae 2022 (1): 1-16. https://doi.org/10.5852/naturae2022a1

\section{RÉSUMÉ}

Le Mercure Arethusana arethusa (Denis \& Schiffermüller, 1775) est un papillon qui, actuellement, n'est pas considéré comme menacé au sein des Listes rouges de France et d'Europe mais dont la distribution apparaît cependant en déclin. Le Conservatoire d'Espaces naturels des Hauts-de-France préserve deux des trois stations restantes en région. Sur la base de captures-marquages-recaptures et d'observations réalisées entre 2013 et 2019 sur les deux dernières populations du département de la Somme, nous avons étudié les paramètres démographiques, la mobilité des individus et leur utilisation des habitats. Les effectifs des populations ont été estimés avec une certaine précision puisque les marges d'erreur des estimations pour un niveau de confiance de $95 \%$ étaient comprises entre 13 et $19 \%$ selon les sites et les années. Des variations importantes d'effectifs, de plus de $50 \%$, ont été relevées entre certaines années et reflètent les tendances observées au niveau national dans le cadre des suivis des communautés de papillons de jours. Durant les trois années de suivis, la taille des populations est la plus élevée entre le 20 août et le 30 août. Le pic de vol des femelles intervient entre trois et six jours après celui des mâles et dure moins longtemps. Selon les sites et les années, la durée de vie moyenne est comprise entre 5,75 et 12 jours. Le sexe ratio mâles/femelles est de 1,18 en 2018 et de 1,28 en 2019. Si le contexte des sites étudiés (faible superficie, isolement) n'a pas permis d'étudier les capacités de dispersion d'A. arethusa, nous avons constaté que l'espèce se déplace peu dans ce contexte. La distance moyenne de déplacement est de $86,46 \pm 6,34$ m après au moins trois recaptures $(n=81)$. La distance maximale relevée entre deux captures est de $333 \mathrm{~m}$. Les pelouses qui présentent une hauteur de végétation rase $(6,57 \pm 2,39 \mathrm{~cm})$, avec des affleurements crayeux $\left(8,21 \pm 7,35 \%\right.$ par $\left.\mathrm{m}^{2}\right)$ et un recouvrement riche en Festuca ovina Gr. $\left(22,6 \pm 9,54 \%\right.$ par $\left.\mathrm{m}^{2}\right)$, constituent les habitats larvaires. Si ces milieux constituent également les habitats préférentiels des adultes, les pelouses plus fermées et jeunes ourlets calcicoles apparaissent complémentaires car elles offrent des ressources nectarifères qui sont faibles sur les pelouses les plus rases en période de vol du papillon. Nos résultats conduisent à faire émerger une question de conservation : comment faire coexister sur des petits espaces des dynamiques spatiales et temporelles de la végétation permettant d'assurer une disponibilité en ressources et en habitats nécessaires à l'accomplissement du cycle de vie d'A. arethusa? Dans des paysages dominés par les grandes cultures, nous soulignons le rôle fonctionnel des prairies, jachères et bandes enherbées pour renforcer les populations en place et reconnecter les espaces fragmentés. 


\begin{abstract}
Ecology and conservation of the False grayling Arethusana arethusa (Denis \& Schiffermüller, 1775) (Butterfly: Satyrinae) in Hauts-de-France.

The False Grayling Arethusana arethusa (Denis \& Schiffermüller, 1775) is a butterfly which is not part of the red list of threatened species in France and Europe however its distribution is declining. The Conservatoire d'espaces naturels des Hauts-de-France preserves two of the three remaining regional locations. Based on capture-mark-release method and observations carried out from 2013 to 2017, we assessed the size of the two last populations of the department of Somme and studied the mobility, resource use and habitat. Mark-release method produce highly accurate estimates. For a 95\% confidence interval, error margin of population size estimates ranged from 13 to 19 percent. Population size estimates showed a high inter-annual variation of more than 50 percent. The same results are observed on a national scale. During the three years of monitoring, the population size reaches its maximum between the $20^{\text {th }}$ and the $30^{\text {th }}$ of august. The peak flight time of females starts three to six days after the males and is shorter. The average adult life span ranged from 5.57 to 12 days. The ratio of males to females is 1.18 in 2018 and 1.28 in 2019. Given the isolation and small size of the study sites, we could not assess dispersal ability of $A$. arethusa. However, the low mobility of $A$. arethusa has been confirmed, with a mean movement distance of $86.46 \pm 6.34 \mathrm{~m}$ over three recapture periods $(\mathrm{n}=81)$. The maximum distance between two captures was $333 \mathrm{~m}$. Shorter grass (Height $=6.57 \pm 2.39 \mathrm{~cm}$ ) with large spaces of bare soil $\left(8.21 \pm 7.35 \%\right.$ per $\left.\mathrm{m}^{2}\right)$ where clumps of Festuca ovina Gr. develops $\left(22.6 \pm 9.54 \%\right.$ cover per $\left.\mathrm{m}^{2}\right)$ is the favorite habitat for larvae. Because these habitats are low flowering in august during the adult flight period, taller forms of grasses seems to be an important nectar resource. A conservation issue is raised: how to manage the vegetation In order to provide nectar resource for the adults and suitable habitat for the larvae of $A$. arethusa in small and isolated areas? In this context, we emphasize the functional part of fallows and grass strips in boosting in situ populations and reuniting scattered areas.

KEY WORDS

Mark-recapture, habitat use, butterfly, calcareous grassland, management.
\end{abstract}

\section{INTRODUCTION}

Située sur la frange nord du Bassin parisien, la région Hautsde-France repose sur des formations sédimentaires calcaires, souvent recouvertes de limons sur les plateaux, mais qui affleurent à la faveur d'accidents topographiques permettant l'expression de pelouses, fourrés et boisements calcicoles. Les pelouses calcicoles des Hauts-de-France, encore appelées «larris» ou "savarts», constituent pour l'essentiel un héritage des premières occupations humaines et d'activités agro-pastorales pluriséculaires (Dutoit 1996). Elles ont acquis au cours du temps une richesse floristique et faunistique à forte valeur patrimoniale. Les modifications des pratiques agricoles au cours du $\mathrm{xx}^{\mathrm{e}}$ siècle, particulièrement marquées en région Hauts-de-France du fait de ses terres limoneuses, ont engendré un bouleversement du paysage rural et une régression spectaculaire des surfaces de pelouses calcicoles. Il est considéré que les habitats semi-naturels de pelouses calcicoles ont, par reconversion ou abandon, régressé de plus de $95 \%$ au cours du Xxe siècle (Boulet 1986). Ils sont aujourd'hui estimés à 9600 ha (GRéB Hauts-de-France 2020). Cette évolution s'est traduite par un appauvrissement et un isolement de la flore et de la faune qu'ils hébergent. $43 \%$ des espèces régionales de Rhopalocères sont associées aux pelouses et $60 \%$ des papillons de jour menacés de l'ex-Picardie sont liés aux pelouses et friches thermophiles (Picardie Nature 2016). La mobilisation de plusieurs acteurs du territoire a permis d'enrayer les pertes d'habitats et de restaurer de nombreuses pelouses calcicoles mais la tendance au déclin se poursuit. Désormais, le changement climatique s'affirme auprès des spécialistes comme une nouvelle menace sur des populations déjà affaiblies et fragmentées, notamment pour les papillons de jour qui présentent le plus souvent de faibles capacités de dispersion (Warren et al. 2001; Settele et al. 2008).

Le Mercure, ou Petit Agreste, Arethusana arethusa (Denis \& Schiffermüller, 1775) (Fig. 1) est un Lépidoptère Satyrinae dont la répartition s'étend d'Europe occidentale jusqu'en Asie. En Europe, les principales populations se localisent en Bulgarie, Espagne, Hongrie, Turquie et dans les pays des Balkans (Van Swaay \& Warren 1999). D'après les mêmes auteurs, les zones occupées en France représenteraient 5 à $15 \%$ de la répartition européenne.

Abondant dans le sud et le centre de l'Europe, $A$. arethusa ne fait pas partie des espèces considérées comme "menacées" dans la Liste rouge européenne (Van Swaay et al. 2010). Cependant, disparu d'Allemagne (Ebert et al. 2008; Reinhardt \& Bolz 2011) et de Suisse (Wermeille et al. 2014), des signes inquiétants de régression s'observent désormais au cœur de son aire de répartition. En Grèce, Zografou et al. (2014) ont corrélé des extinctions locales de population d'A. arethusa à l'élévation récente des températures. Cette étude semble confirmer les prévisions de Settele et al. (2008) qui soulignent la forte sensibilité de l'espèce aux changements climatiques. Pour ces auteurs, la répartition européenne d'A. arethusa pourrait régresser de 65 à $95 \%$ d'ici 2080 conformément aux différents scénarii européens d'élévation des températures.

En France, $A$. arethusa ne figure pas non plus parmi les espèces menacées de la Liste rouge des Rhopalocères de France métropolitaine (UICN France et al. 2014). Pourtant, ses populations ne semblent prospérer que dans le domaine 


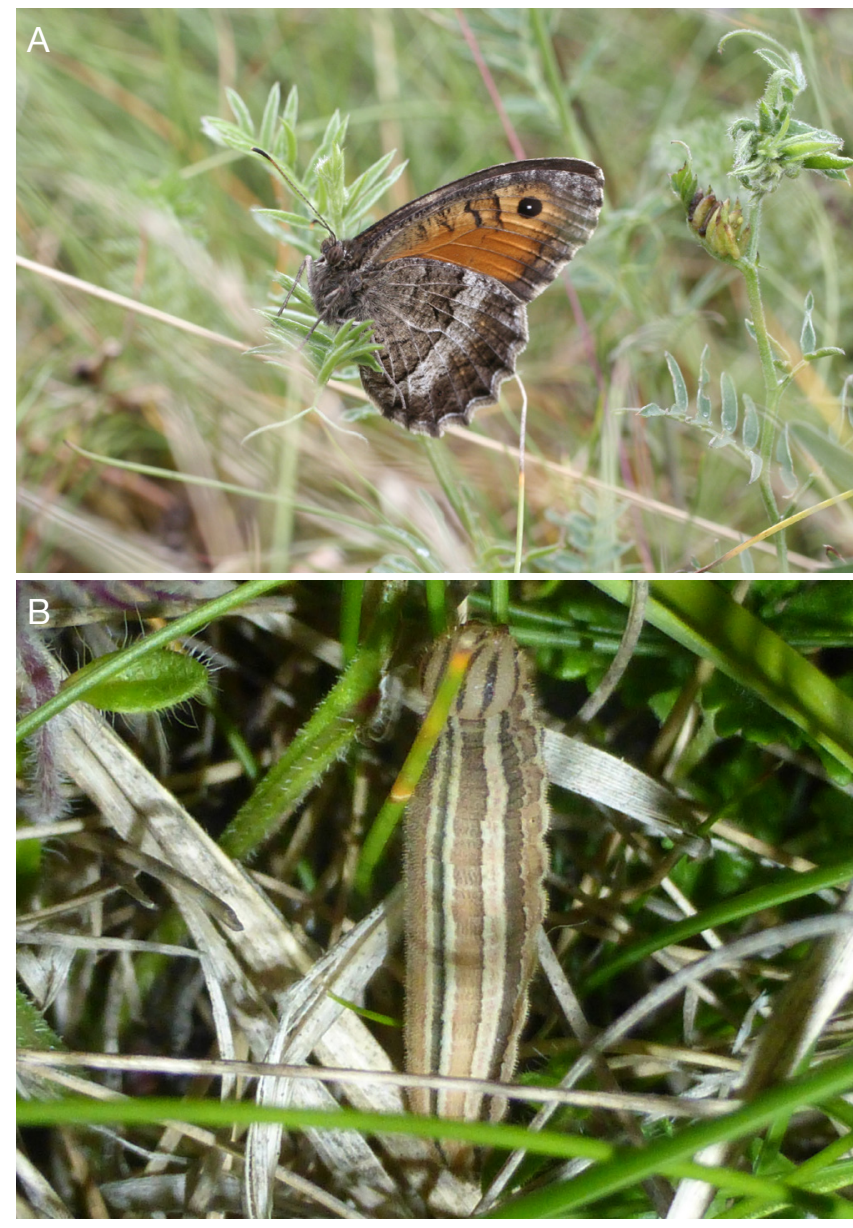

FIG. 1. - Le Mercure Arethusana arethusa (Denis \& Schiffermüller, 1775) posé sur une vesce (A) et chenille de Mercure sur une tige de fétuque (B). Crédits photos: Damien Top (A) et Guillaume Meire (B).

méditerranéen. En effet, ce n'est qu'en Occitanie et en ProvenceAlpes-Côte d'Azur que l'espèce n'est pas considérée comme menacée (Bence 2014 ; Louboutin et al. 2019). Plus au nord, $A$. arethusa est "quasi-menacé» sur le territoire Rhône-alpin (Baillet \& Guicherd 2018) et en «danger critique d'extinction» en Auvergne (Bachelard \& Fournier 2013). Éteinte en Alsace (IMAGO 2014) et en "danger critique d'extinction" en Franche-Comté (Jugan et al. 2013), l'espèce est considérée comme "vulnérable» en Bourgogne (Ruffoni 2015) et est inscrite sur la Liste rouge des insectes de l'ancienne région Champagne-Ardenne (Coppa et al. 2007). Dans le domaine atlantique, $A$. arethusa a toujours été noté comme étant "plutôt rare et localisé» par les différents lépidoptéristes l'observant. Il est «quasi-menacé» en Haute-Normandie (Lorthiois 2015), «vulnérable» en région Centre (Lett et al. 2007) et est «en danger» en Aquitaine (OAFS 2019), Poitou-Charentes (Poitou-Charentes Nature 2019) ainsi qu'en Île-de-France (Dewulf \& Houard 2016).

Cité une seule fois en Nord-Pas-de-Calais au XIx ${ }^{\mathrm{e}}$ siècle (François 2003), A. arethusa atteint aujourd'hui sa limite septentrionale sur le territoire de la Picardie où il demeure très rare et a été récemment évalué en "danger d'extinction»

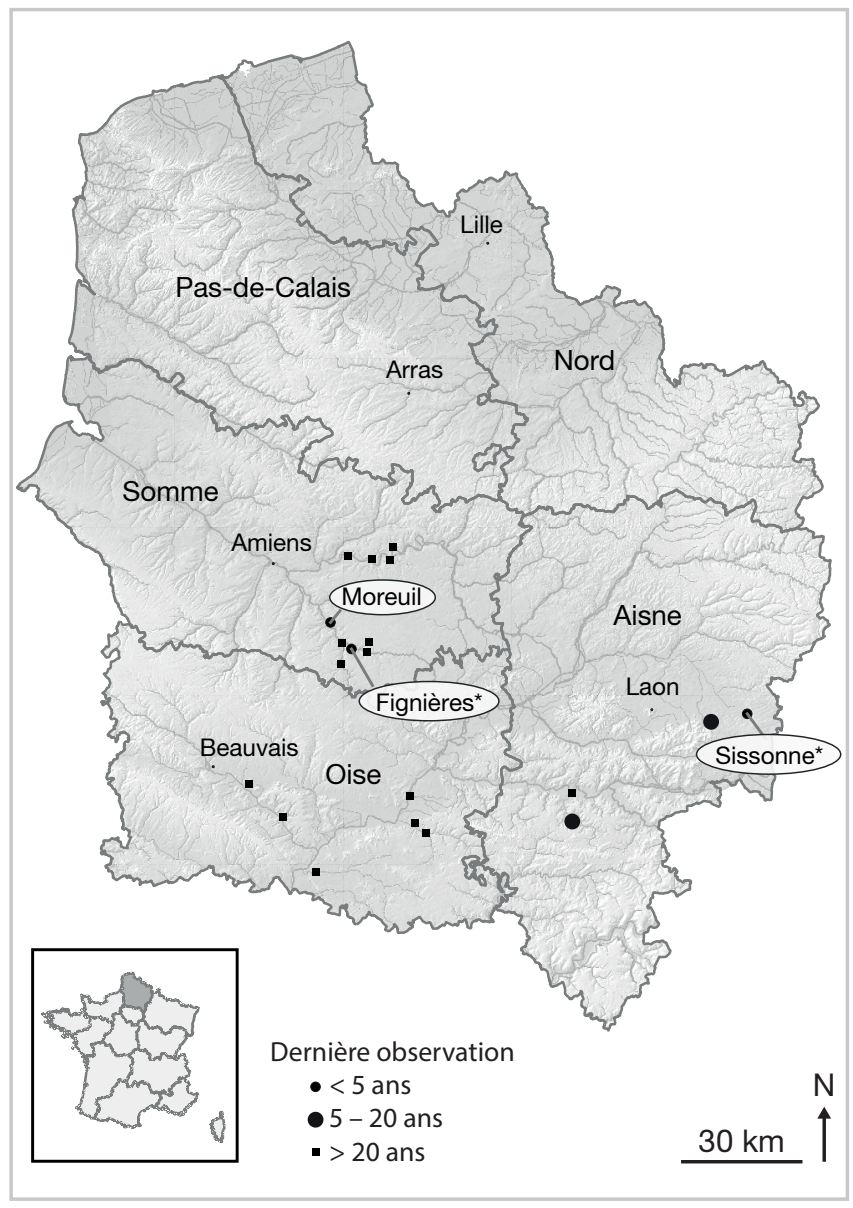

FIG. 2. - Répartition d'Arethusana arethusa (Denis \& Schiffermüller, 1775) en Hauts-de-France. Les observations antérieures au xxe siècle ne sont pas présentées. Sources des données: ADEP, Picardie Nature, Conservatoire d'Espaces naturels Hauts-de-France. * , site géré par le Conservatoire d'Espaces naturels.

(Picardie Nature 2016). Son aire d'occupation s'est réduite de plus de $80 \%$ en 30 ans (Fig. 2). Dans ce contexte, la conservation des populations d'A. arethusa revêt un enjeu prioritaire pour le Conservatoire d'Espaces naturels des Hauts-de-France (CEN Hauts-de-France par la suite) qui préserve deux des trois dernières populations régionales connues.

Devant les enjeux de conservation, un suivi démographique quantitatif, basé sur la méthode de "capture-marquagerecapture", a été initié en 2013 et renouvelé en 2018 puis 2019 sur les deux dernières populations du département de la Somme. En parallèle, il nous était nécessaire de mieux comprendre les facteurs déterminant la répartition de l'espèce sur les sites pour pouvoir mettre en place des actions conservatoires appropriées.

Cet article décrit la structure démographique (taille de la population, survie, durée de vie) de deux populations d'A. arethusa. Les déplacements (intra-site) et l'utilisation des milieux par les imagos sont étudiés (habitats préférentiels, plantes nectarifères). Une caractérisation des habitats larvaires est également proposée. Les résultats sont ensuite confrontés aux connaissances disponibles afin de discuter des orientations d'actions conservatoires. 
TABLEAU 1. - Description succincte des quatre habitats herbacés relevés sur les sites de Fignières (FIG) et de Moreuil (MRL) et proportion de ces habitats sur les deux sites (en \%). D'après l'inventaire et la cartographie des végétations des sites (Meire \& Rivière 2015, 2016).

\begin{tabular}{|c|c|c|c|c|}
\hline \multirow[b]{2}{*}{ Habitat } & \multirow[b]{2}{*}{ Description succinte } & \multicolumn{3}{|c|}{$\begin{array}{l}\text { Répartition des habitats sur } \\
\text { l'aire d'étude (\%) }\end{array}$} \\
\hline & & FIG & MRL & $\mathrm{FIG}+\mathrm{MRL}$ \\
\hline $\begin{array}{l}\text { Pelouse juvénile } \\
\text { à mature (PJM) }\end{array}$ & $\begin{array}{l}\text { Végétation basse }(<10 \mathrm{~cm} \text { ) présentant de nombreuses écorchures ( } \geq 10 \%) \text {. } \\
\left.\text { Cortège floristique diversifié ( }>20 \text { espèces } / \mathrm{m}^{2}\right)\end{array}$ & 35,5 & 17,5 & 23 \\
\hline $\begin{array}{l}\text { Pelouse mature } \\
\text { à sénescente (PMS) }\end{array}$ & $\begin{array}{l}\text { Hauteur de végétation comprise entre } 10 \text { et } 30 \mathrm{~cm} \text {. } \\
\text { Affleurement crayeux localisé. } \\
\text { Cortège floristique diversifité (15-20 espèces/m²). } \\
\text { Les pelouses sénescentes sont marquées par l'apparition d'espèces } \\
\text { sociales: Brachypodium pinnatum (L.) P.Beauv., Bromopsis erecta (Huds.) } \\
\text { Fourr., Carex flacca Schreb. }\end{array}$ & 31 & 5,5 & 13 \\
\hline $\begin{array}{l}\text { Ourlet juvénile } \\
\text { à mature (OJM) }\end{array}$ & $\begin{array}{l}\text { Formation dense, de } 40 \mathrm{~cm} \text { de hauteur en moyenne, présentant une } \\
\text { certaine homogénéité floristique }\left(10-15 \text { espèces } / \mathrm{m}^{2}\right) \text {. } \\
\text { Brachypodium pinnatum et/ou Bromopsis erecta dominant. }\end{array}$ & 26 & 37,5 & 34 \\
\hline $\begin{array}{l}\text { Ourlet mature } \\
\text { à sénescent (OMS) }\end{array}$ & $\begin{array}{l}\text { Formation dense de } 40 \mathrm{~cm} \text { de hauteur en moyenne. } \\
\left.\text { Faible diversité floristique (<10 espèces } / \mathrm{m}^{2}\right) \text {. } \\
\text { Brachypodium pinnatum et/ou Bromopsis erecta dominant. } \\
\text { Apparition des ligneux }\end{array}$ & 7,5 & 39,5 & 30 \\
\hline
\end{tabular}

\section{MATÉRIELS ET MÉTHODES}

\section{SiTES D'ÉTUDES}

Les sites d'études sont localisés en région Hauts-de-France, au sud-est du département de la Somme, sur les communes

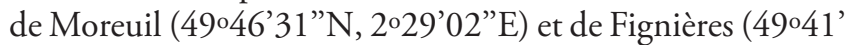
$\left.05^{\prime} \mathrm{N}, 2035^{\prime} 17^{\prime} \mathrm{E}\right)$. Reposant sur de la craie du Crétacé supérieur, les sites abritent des pelouses calcaricoles secondaires endémiques du Nord de la France qui, d'un point de vue phytosociologique, se rattachent à la série de végétation de l'Avenulo pratensis-Festucetum lemanii Boullet et al. 1984 (Code UE: 6210-22, EUNIS: E1.26) (Tableau 1).

Le site de Moreuil ne bénéficie pas de gestion conservatoire. Il se compose d'une pelouse calcaricole de 2,5 ha dont les conditions stationnelles (forte pente, sol maigre, exposition sud) freinent la dynamique naturelle de la végétation (Fig. 3A). Ces conditions associées à la forte pression de broutage et de grattage par les lapins permettent le développement de pelouses rases riches en affleurements crayeux, dites «écorchées cuniculogènes». Une prairie sèche de 3,3 ha surplombe la pelouse. En place pour moitié depuis plus de 20 ans, elle se compose d'un ourlet calcicole du Trifolion medii T. Müll. Déclarée en jachère jusqu'en 2014, une fauche automnale (novembre) y était réalisée. Depuis la réforme de la politique agricole commune (PAC) de 2015, elle est déclarée en prairie temporaire et est fauchée en juillet pour produire du foin.

Le site de Fignières abrite un complexe de pelouse calcicole de près de trois hectares (Fig. 3B). Classé par Arrêté préfectoral de Protection de Biotope (APPB), il est géré depuis 2001 par le CEN Hauts-de-France. L'abandon du pâturage dans les années 1960 a nécessité d'importants travaux de déboisement entre 2005 et 2009 pour restaurer les surfaces de pelouses historiques. Afin de diversifier les végétations herbacées et étendre les pelouses rases et écorchées, des lapins ont en parallèle été réintroduits pour renforcer la population du site et accroître les pressions d'herbivorie. Si l'opération s’est avérée pertinente, elle n’a pu être pérennisée à la suite des dégâts occasionnés sur les cultures riveraines. Depuis, des fauches avec exportation des rémanents sont régulièrement réalisées pour limiter la progression des graminées sociales et des fourrés arbustifs. Depuis 2019, les pelouses les plus dégradées sont gérées par pâturage mixte ovin/caprin extensif (0,25-0,35 unités gros bétail [UGB]/ha/an).

Les deux sites, distants de huit kilomètres environ, s'intègrent dans une matrice paysagère peu propice à la dispersion des individus puisque dominée par les grandes cultures. Les deux stations d'A. arethusa semblent isolées. Aucun individu n'a jamais été observé sur les milieux herbacés interstitiels au cours des campagnes de prospection $(2010,2013,2015,2018$, 2019) et aucun échange d'individus n'a été mis en évidence lors des opérations de capture-marquage-recapture.

\section{ESPÈCE ÉTUDIÉE}

A. arethusa est une espèce monovoltine qui hiverne au premier stade larvaire. La femelle laisse tomber les œufs un à un dans la végétation (Bink 1992; Fartmann \& Hermann 2006). Les chenilles se nourrissent de diverses poacées (Bromus sp., Festuca sp., Datylis sp. et Poa sp.) (Bink 1992). Actives de jour pendant les deux premiers stades, elles ne s'alimentent plus que la nuit au cours des deux derniers (Geiger 1987). La chrysalide repose dans le sol, 2 à $3 \mathrm{~cm}$ sous la surface et le papillon émerge après 4 à 5 semaines (Lafranchis et al. 2015). La période de vol s'étend de juillet à septembre avec des mâles volant plus précocement que les femelles. Le sexe ratio serait en faveur des mâles selon les observations de plusieurs auteurs (Pintureau 1977).

A. arethusa se rencontre principalement au sein des pelouses calcicoles et siliceuses sèches mais est également cité en situation de clairière forestière et de prairie mésophile (Van Swaay et al. 2006). En région Hauts-de-France, l'espèce fréquente les milieux les plus xériques, conditionnés par un sol très filtrant ou par une forte pente: pelouses sur craie, pelouses et friches sur sables calcaires mais également certaines clairières xérothermophiles de hêtraies calcicoles. 

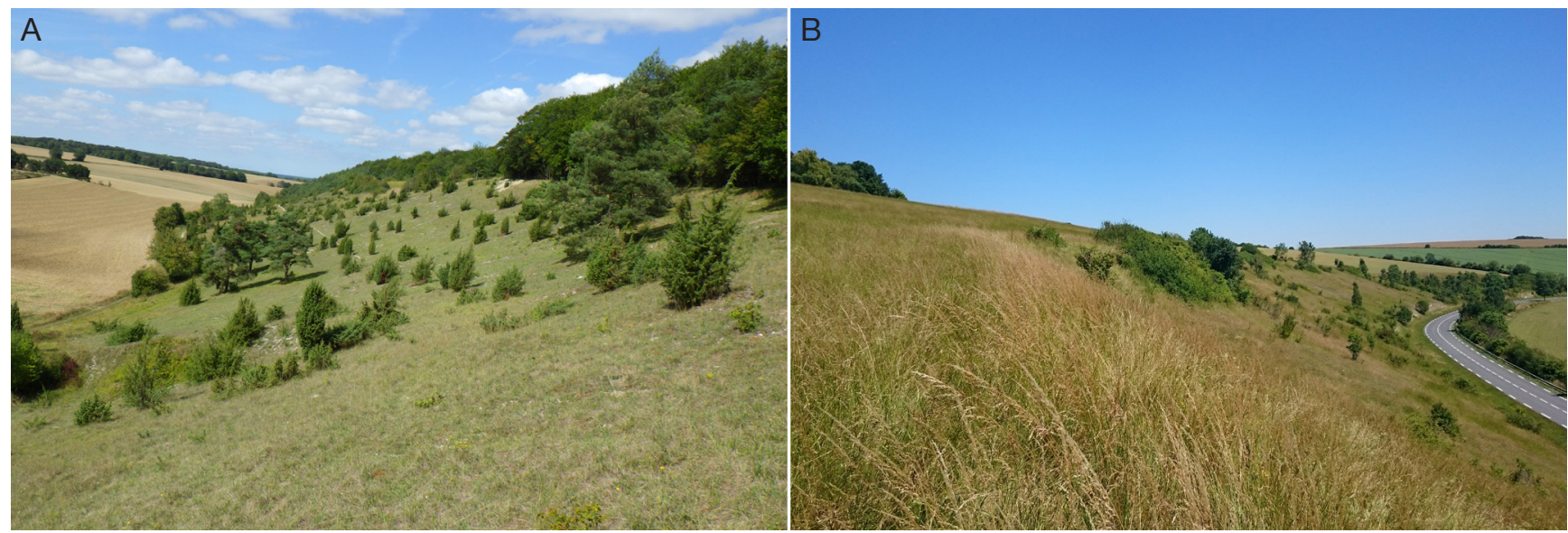

FIG. 3. - Vue de la pelouse de Moreuil (A) et de Fignières (B). Crédits photos: G. Meire.

TABLEAU 2. - Dates de suivis et nombre d'individus d'Arethusana arethusa (Denis \& Schiffermüller, 1775) capturés et recapturés sur les sites de Moreuil (MRL) en 2013 et 2018 et de Fignières (FIG) en 2013, 2018 et 2019. Les sexes n'ont pas été relevés en 2013.

\begin{tabular}{|c|c|c|c|c|c|c|c|c|c|c|c|c|c|}
\hline \multirow{3}{*}{$\frac{\text { Année }}{2013}$} & \multirow{4}{*}{$\begin{array}{l}\text { Date de suivi } \\
\begin{array}{l}14,19,20,23,28 \\
\text { et } 30 \text { août }\end{array}\end{array}$} & \multicolumn{6}{|c|}{ MRL } & \multicolumn{6}{|c|}{ FIG } \\
\hline & & \multicolumn{3}{|c|}{ Capture } & \multicolumn{3}{|c|}{ Recapture } & \multicolumn{3}{|c|}{ Capture } & \multicolumn{3}{|c|}{ Recapture } \\
\hline & & \multirow{2}{*}{$\begin{array}{c}\text { Mâle } \\
-\end{array}$} & \multicolumn{2}{|c|}{ Femelle Total } & \multirow{2}{*}{$\begin{array}{c}\text { Mâle } \\
-\end{array}$} & \multicolumn{2}{|c|}{ Femelle Total } & \multirow{2}{*}{$\begin{array}{c}\text { Mâle } \\
-\end{array}$} & \multicolumn{2}{|c|}{ Femelle Total } & \multirow{2}{*}{$\begin{array}{c}\text { Mâle } \\
-\end{array}$} & \multicolumn{2}{|c|}{ Femelle Total } \\
\hline 2013 & & & - & 902 & & - & 132 & & - & 356 & & - & 94 \\
\hline 2018 & $\begin{array}{l}6,8,10,14,17,21 \\
23 \text { et } 28 \text { août }\end{array}$ & 230 & 94 & 324 & 81 & 41 & 122 & 126 & 69 & 195 & 121 & 23 & 144 \\
\hline 2019 & $\begin{array}{l}13,16,20,23,26,29 \\
\text { août et } 2 \text { septembre }\end{array}$ & - & - & - & - & - & - & 102 & 80 & 182 & 70 & 24 & 94 \\
\hline
\end{tabular}

\section{MÉTHOdE}

\section{Capture-marquage-recapture}

La méthode de "capture-marquage-recapture» (CMR) a été utilisée pour étudier les populations d'A. arethusa. Elle a été préférée à d'autres protocoles plus largement utilisés dans le cadre d'estimation d'abondance de papillons de jours (Pollard Walks [Pollard 1977; Thomas 1983, 1991; Haddad et al. 2008], Distance sampling [Buckland et al. 2001]) car elle permet également d'estimer des paramètres démographiques tels la survie et le recrutement mais aussi de caractériser les déplacements et l'utilisation des milieux par les individus.

Sur les deux sites, six sessions de capture ont été réalisées entre le 14 et le 30 août 2013 et huit sessions entre le 6 et le 28 août 2018. En 2019, en l'absence d'autorisation pour le site de Moreuil, seul le site de Fignières a été suivi entre le 13 août et le 2 septembre (Tableau 2).

Les sessions de CMR ont été réalisées avec des intervalles de deux à cinq jours dans de bonnes conditions météorologiques (ciel dégagé, vent faible et températures supérieures à $20^{\circ} \mathrm{C}$ ), entre $10 \mathrm{~h}$ et $17 \mathrm{~h}$. Ils duraient $3 \mathrm{~h} \pm 30$ minutes sur le site de Moreuil ( $30 \mathrm{~min} / \mathrm{ha}$ ) et $1 \mathrm{~h} 40 \pm 15$ minutes sur le site de Fignières (30 $\mathrm{min} / \mathrm{ha})$ de manière à parcourir l'ensemble des espaces herbacés. Chaque individu nouvellement capturé a été marqué d'un numéro d'identification unique avec un feutre permanent pigmenté à base d'eau (Uni POSCA PC-3M) sur le dessous de l'aile postérieure, puis relâché (Fig. 4). Les individus recapturés dans la journée du marquage n’ont pas été pris en compte. L'emplacement des captures et recaptures a été relevé à l'aide d’un GPS ( $\pm 3 \mathrm{~m}$ de précision). Les sexes ont été différenciés lors des suivis menés en 2018 et 2019 mais pas en 2013.

\section{Points d'observation}

Trois points d'observation ont été positionnés sur le site de Fignières en 2015 et en 2018 pour étudier les plantes nectarifères préférentiellement visitées par $A$. arethusa. Pour ne pas influencer les comportements, les points d'observation ont été positionnés en haut de coteau et les observations se sont faites à la jumelle dans un rayon de 30 mètres autour des points d'observations. Ce positionnement a permis de couvrir $40 \%$ de l'aire de présence d'A. arethusa $\left(6100 \mathrm{~m}^{2}\right)$ du site et d'étudier les comportements dans un échantillon d'habitats représentatif du site (pelouse pionnière à mature : $34 \%$; pelouse mature à sénescente : $53 \%$; ourlet : $13 \%)$. Cinq sessions d'observations avec des intervalles de 4-5 jours ont été réalisées entre le 14 août et le 8 septembre 2015, six sessions ont été reconduites entre le 8 et 28 août 2018. Parce qu' $A$. arethusa butine davantage le matin et en fin d'après-midi (Lafranchis 2001; Lafranchis et al. 2015), les observations ont été réalisées par temps chaud et ensoleillé, entre $10 \mathrm{~h}$ et $12 \mathrm{~h}$ ou entre $16 \mathrm{~h}$ et $17 \mathrm{~h}$ et duraient 20 minutes. 


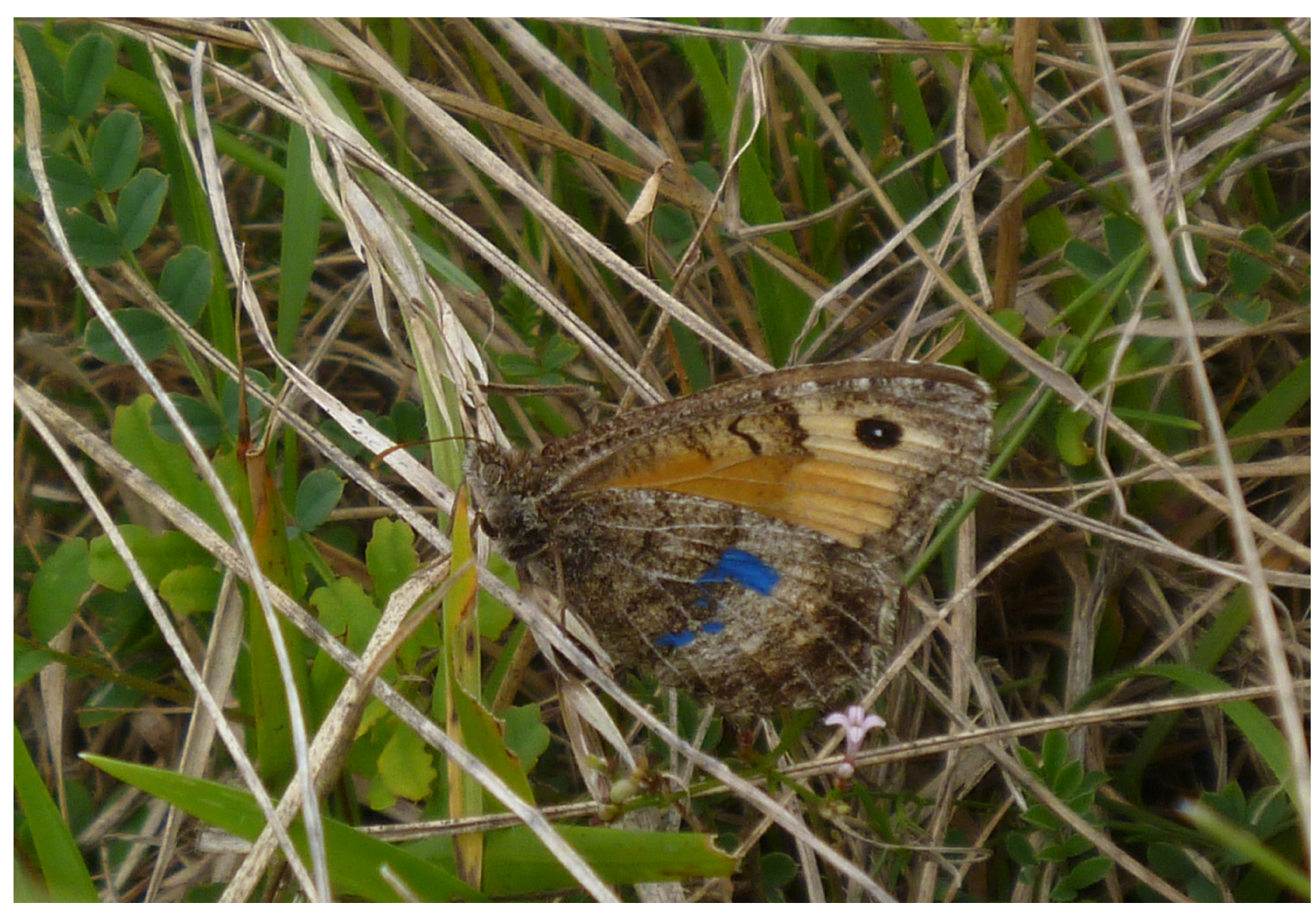

FIG. 4. - Arethusana arethusa (Denis \& Schiffermüller, 1775) marqué sur le dessous de l'aile postérieure. Crédit photo: G. Meire.

\section{Recherche de chenille}

Afin de caractériser les habitats larvaires, des prospections à vue de chenilles ont été réalisées les 20 juin 2018 et 6 juillet 2018 sur le site de Moreuil, et le 27 juin 2018 sur le site de Fignières, entre 21 h30 et 3 h00. Des recherches de chenilles ont été effectuées au sein de 180 placettes de $1 \mathrm{~m}^{2}$ tirées de manière aléatoire au sein des différents habitats herbacés des sites: pelouse juvénile à mature $(\mathrm{n}=56)$, pelouse mature à sénescente $(n=64)$, ourlet juvénile à mature $(n=60)$. L'échantillonnage a été réalisé sur l'aire d'occupation d'A. arethusa relevée en 2013 (3,8 ha). Pour chaque quadrat, le recouvrement de sol à nu, celui de la fétuque et la hauteur moyenne de la végétation ont été estimés. En parallèle, trois à cinq observateurs selon les dates ont recherché des chenilles de manière opportuniste en ciblant préférentiellement les pelouses abritant des fétuques. Pour chaque chenille trouvée, la plante hôte a été déterminée et les paramètres cités précédemment ont été relevés dans des placettes de $1 \mathrm{~m}^{2}$.

\section{ANALYSE DES DONNÉES}

DE «CAPTURE MARQUAGE RECAPTURE» (CMR)

Le programme POPAN pour population ouverte intégré dans le programme MARK 7.0 (White \& Burnham 1999) a été utilisé pour estimer la taille totale $(\mathrm{N})$ et quotidienne $(\mathrm{Ni})$ des populations.
Les probabilités de survie ( $\varphi \mathrm{i})$, de capture (pi) et le taux de recrutement (Penti) journalier ont été estimés. Plusieurs modèles ont été testés en ajoutant des covariables (temps [t], sexe $[\mathrm{g}])$ sur les paramètres de survie ( $\varphi \mathrm{i})$ et de capture (pi). Nous avons testé l'addition du sexe et du temps $(\mathrm{g}+\mathrm{t})$ ainsi que le sexe $(\mathrm{g})$ et le temps $(\mathrm{t})$ indépendamment ou comme paramètres constants (.).

Le meilleur modèle a été sélectionné sur la base de l'Akaike's Information Criterion (AIC) (Burnham \& Anderson 2002). Dans le cas de modèles proches $(\triangle \mathrm{AIC}<2)$, nous avons retenu celui incluant le moins de paramètres pour estimer les probabilités de capture et de survie et nous avons utilisé une procédure de "model averaging" pour estimer la taille totale et journalière des populations (Burnham \& Anderson 2002).

La durée de vie moyenne a été estimée à partir du taux de survie journalier selon la formule $\hat{e}=(1-\varphi)-1-0,5$ (Nowicki et al. 2005).

Les localisations des événements des captures/recaptures ont été utilisées pour analyser les déplacements d'A. arethusa. Pour chaque individu, la distance euclidienne entre chaque capture est calculée avec QGIS 2.18.14 (QGIS Development Team 2016). Pour les individus capturés au moins trois fois, la distance entre les deux points de capture les plus éloignés est mesurée et une estimation de la taille des surfaces fréquentées est calculée en utilisant la méthode MCP ou "polygone 
TABLEAU 3. - Présentation des meilleurs modèles POPAN selon l'Akaike information criterion (avec $\Delta$ AICc $<2$ ), estimation des probabilités de capture et de

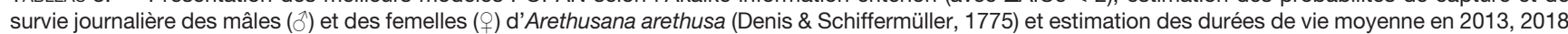
et 2019 sur les sites de Fignières (FIG) et Moreuil (MRL). Paramètres des modèles: $\Delta$ AICc, différence d'AlCc par rapport au meilleur modèle; $\varphi$, probabilité de survie journalière; Nb. Par., nombre de paramètres; $\mathbf{p}$, probabilité de capture; pent, recrutement. Les intervalles de confiance à $95 \%$ sont donnés entre parenthèses. ${ }^{*}$, lorsque la probabilité de survie varie en fonction du temps, une moyenne est donnée à partir du meilleur avec $\varphi$ constant.

\begin{tabular}{|c|c|c|c|c|c|c|c|c|c|c|c|}
\hline \multirow[b]{2}{*}{ Site } & \multirow[b]{2}{*}{ Année } & \multirow[b]{2}{*}{ Modèle } & \multirow[b]{2}{*}{$\triangle \mathrm{AICc}$} & \multirow[b]{2}{*}{ Nb. Par } & \multicolumn{3}{|c|}{ Probabilité de capture (p) } & \multicolumn{3}{|c|}{ Probabilité de survie $(\varphi)$} & \multirow{2}{*}{$\begin{array}{l}\text { Durée de } \\
\text { vie } \\
\text { (jours) }\end{array}$} \\
\hline & & & & & $\hat{0}$ & 우 & o우 & $\sigma^{\pi}$ & 우 & jo & \\
\hline \multirow[t]{3}{*}{ FIG } & 2013 & $\{p(),. \varphi(),. \operatorname{pent}(t)\}$ & 0 & 8 & - & - & $\begin{array}{c}0,29 \\
(0,21-0,37)\end{array}$ & - & - & $\begin{array}{c}0,87 \\
(0,82-0,92)\end{array}$ & $\begin{array}{c}7,19 \\
(5,06-12,00)\end{array}$ \\
\hline & 2018 & $\left\{\mathrm{p}(\mathrm{g}), \varphi(\mathrm{t}), \operatorname{pent}\left(\mathrm{g}^{*} \mathrm{t}\right)\right\}$ & 0 & 23 & $\begin{array}{c}0,44 \\
(0,36-0,52)\end{array}$ & $\begin{array}{c}0,30 \\
(0,19-0,43)\end{array}$ & - & $\begin{array}{c}0,91^{*} \\
(0,73-0,97)\end{array}$ & $\begin{array}{c}0,91^{*} \\
(0,73-0,97)\end{array}$ & - & $\begin{array}{c}10,61 \\
(3,20-32,83)\end{array}$ \\
\hline & 2019 & $\left\{p(g), \varphi(t)\right.$, pent $\left.\left(g^{*} t\right)\right\}$ & 1,98 & 16 & $\begin{array}{c}0,43 \\
(0,27-0,59)\end{array}$ & $\begin{array}{c}0,36 \\
(0,29-0,45)\end{array}$ & - & $\begin{array}{c}0,92^{*} \\
(0,72-0,96)\end{array}$ & $\begin{array}{c}0,92^{\star} \\
(0,72-0,96)\end{array}$ & - & $\begin{array}{c}12 \\
(3,07-24,50) \\
\end{array}$ \\
\hline \multirow[t]{2}{*}{ MRL } & 2013 & $\{p(),. \varphi(),. \operatorname{pent}(t)\}$ & 1,80 & 8 & - & - & $\begin{array}{c}0,15 \\
(0,10-0,21)\end{array}$ & - & - & $\begin{array}{c}0,84 \\
(0,80-0,88)\end{array}$ & $\begin{array}{c}5,75 \\
(4,5-7,83)\end{array}$ \\
\hline & 2018 & $\left\{\mathrm{p}(),. \varphi(\mathrm{g})\right.$, pent $\left.\left(\mathrm{g}^{\star} \mathrm{t}\right)\right\}$ & 0 & 17 & $\begin{array}{c}0,29 \\
(0,22-0,38)\end{array}$ & $\begin{array}{c}0,29 \\
(0,22-0,38)\end{array}$ & - & $\begin{array}{c}0,81 \\
(0,77-0,85)\end{array}$ & $\begin{array}{c}0,89 \\
(0,84-0,94)\end{array}$ & - & $\begin{array}{c}6,16 \\
(4,50-8,59)\end{array}$ \\
\hline
\end{tabular}

convexe minimum» (White \& Garrot 1990). Parce que nous ne disposons pas des informations sur les mouvements des individus entre les captures, les distances mesurées et les surfaces estimées doivent être considérées comme étant des minima. Les tests non paramétriques de Kruskall-Wallis (test $\mathrm{H}$ ) et de Mann-Whitney (test U) ont été utilisés pour comparer les distances de déplacement entre les sites, les années et les sexes. Les surfaces utilisées par les individus selon les années ont aussi été comparées.

L'analyse de la sélection d'habitat par les imagos d' $A$. arethusa a été effectuée en utilisant la méthode des ratios de sélection (Manly et al. 2002). Pour chacun des quatre habitats herbacés identifiés, un ratio de sélection (RS) a été calculé en divisant le ratio d'utilisation ( $\mathrm{U}$ i) par le ratio de disponibilité (D i), tel que RS i = U i/D i. Sous l'hypothèse d'une absence de sélection de l'habitat i, l'indice de sélectivité RS i devrait être égal à 1 . RS i est supérieur à 1 si l'habitat est préféré, et inférieur à 1 s'il est évité.

Pour étudier les micro-habitats préférentiels des chenilles, le test non paramétrique de Mann-Whitney (test U) a été utilisé pour savoir s'il existe des différences significatives entre les placettes occupées et inoccupées pour les quatre paramètres relevés (hauteur de la végétation, recouvrement de sol à nu, recouvrement de la strate herbacée, recouvrement de la fétuque).

\section{RÉSULTATS}

\section{PARAMÈTRES DÉMOGRAPHIQUES}

En 2013, 1258 individus ont été marqués sur les deux sites. Parmi eux, 226 individus ont été recapturés au moins une fois. En 2018, ce sont 519 individus correspondant à 356 mâles et 163 femelles qui ont été capturés. 266 individus ont été recapturés. 182 individus (102 mâles et 80 femelles) ont été capturés en 2019 sur le site de Fignières et 94 d'entre-deux ont fait l'objet d'une recapture (Tableau 2).

Pour les deux sites et quelles que soient les années, la probabilité de capture (p) ne varie pas en fonction du temps (Tableau 3). Sur le site de Fignières, elle est de 0,44 (IC 95\%
TABLEAU 4. - Taille de population d'Arethusana arethusa (Denis \& Schiffermüller, 1775) estimée d'après les meilleurs modèles POPAN par année et par site. Abréviations: $\delta$, estimation de la taille de la population totale; 0 , estimation de la taille de la population de mâles; + , estimation de la taille de la population de femelles; FIG, Fignières; MRL, Moreuil. Les sexes n'ont pas été différenciés en 2013. Les intervalles de confiance à $95 \%$ sont donnés entre parenthèses.

\begin{tabular}{lcccc}
\hline & & \multicolumn{3}{c}{ Taille de population (N) } \\
\cline { 3 - 5 } Site & Année & $\delta$ & $q$ & $\delta q$ \\
\hline FIG & 2013 & - & - & 731 \\
& 2018 & 174 & 147 & $(600-862)$ \\
& & $(149-199)$ & $(96-198)$ & $(264-370)$ \\
& 2019 & 188 & 146 & 345 \\
& & $(130-246)$ & $(120-172)$ & $(283-423)$ \\
\hline MRL & 2013 & - & - & 3952 \\
& 2018 & 469 & 208 & $(3197-4707)$ \\
& & $(383-555)$ & $(150-266)$ & $(598-797)$ \\
\hline
\end{tabular}

[0,36-0,52]) en 2018 et 0,43 (IC 95\% [0,27-0,59]) en 2019 chez les mâles contre respectivement 0,30 (IC $95 \%[0,19$ $0,43]$ ) et 0,36 (IC $95 \%[0,29-0,45]$ ) chez les femelles. Les valeurs moyennes sont proches de la probabilité de capture relevée en 2013 qui était estimée à 0,29 (IC $95 \%$ [0,21-0,37]). Sur le site de Moreuil, la probabilité de capture ne varie pas avec le sexe. Elle est deux fois plus importante en 2018 qu'en 2013. Estimée à 0,15 (IC $95 \%[0,10-0,21])$ en 2013, elle est en 2018 de 0,29 (IC $95 \%$ [0,22-0,38]).

La probabilité de survie journalière varie peu entre les années au sein d'un même site (Tableau 3). Sur le site de Fignières, elle n'est pas différente entre les sexes mais varie dans le temps en 2018 et 2019. Estimée à 0,87 (IC $95 \%$ $[0,82-0,92])$ en 2013 , la survie journalière moyenne est de 0,91 (IC $95 \%[0,73-0,97]$ ) en 2018 et de 0,92 (IC 95\% $[0,72-0,96])$ en 2019.

Sur le site de Moreuil, la probabilité de survie journalière est de 0,84 (IC $95 \%[0,80-0,88]$ ) en 2013. En 2018, elle est de 0,81 (IC $95 \%[0,77-0,85]$ ) chez les mâles et 0,89 (IC $95 \%[0,84-0,94])$ chez les femelles. 


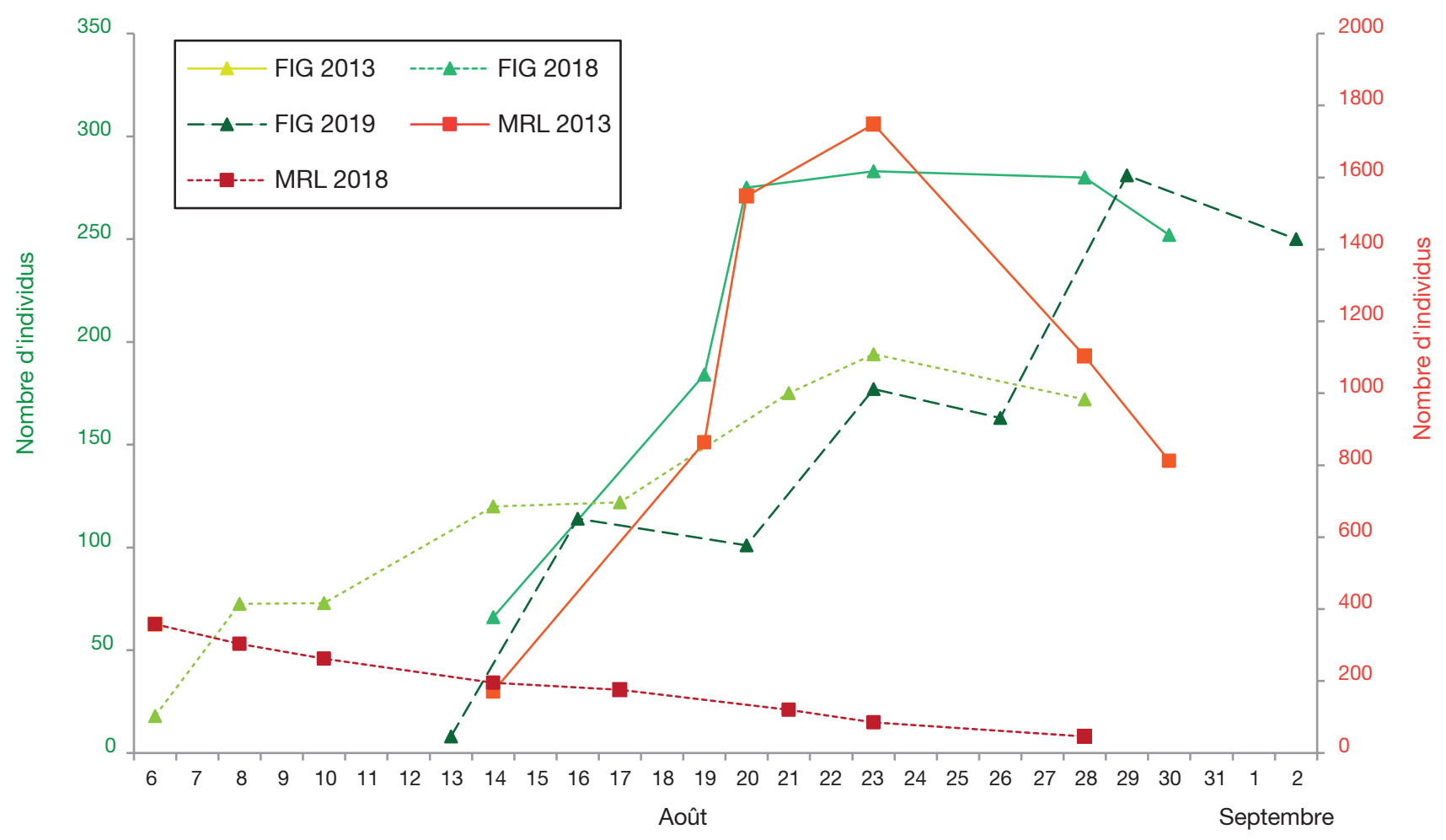

FIG. 5. - Taille de population journalière d'Arethusana arethusa (Denis \& Schiffermüller, 1775) sur les sites de Fignières (FIG; axe principal) et de Moreuil (MRL; axe secondaire) selon les années. Les estimations sont issues des données de capture-marquage-recapture analysées par le modèle POPAN dans le programme MARK. Les modèles utilisés sont présentés dans le Tableau 3.

Selon les sites et les années, la durée de vie moyenne est comprise entre 5,75 et 12 jours (Tableau 3 ). Elle peut atteindre 32,83 jours si l'on prend les bornes des intervalles de confiance à $95 \%$. Le plus grand intervalle de temps relevé entre deux captures est de 20 jours et concerne les deux sexes. Cet intervalle de temps a été relevé au cours des suivis menés en 2018 et 2019.

En 2018, les estimations d'effectifs des populations des deux sites d'études sont significativement inférieures à celles estimées en 2013. Estimée à 3952 individus (IC $95 \%$ [3197-4707]) en 2013, la population du site de Moreuil était estimée à 690 individus (IC 95 \% [598-797]) en 2018, correspondant à une baisse de $83 \%$ des effectifs. À Fignières, la taille de la population est estimée à 312 individus (IC $95 \%$ [264-370]) en 2018 contre 731 individus (IC $95 \%$ [600-862]) en 2013 (-55\%). Elle est restée stable entre 2018 et 2019 (Tableau 4).

Durant les trois années de suivis, le pic de vol se situe entre le 20 et le 30 août (Fig. 5). Un décalage phénologique s'observe entre les années. En 2019, le pic de vol est plus tardif (29 août) tout comme l'apparition des premiers imagos. En 2018, l'évolution de la taille de la population de Moreuil au cours de l'été est particulière puisqu'une baisse progressive des effectifs est observée dès le début du mois d'août.

Sur le site de Fignières, le sexe ratio mâles/femelles est de 1,18 en 2018 et de 1,28 en 2019 (Tableau 4).

Les mâles volent plus précocement que les femelles (Fig. 6). En 2018, sur le site de Fignières, le pic de vol des mâles se situe entre le 10 et le 21 août tandis que celui des femelles est relevé le 23 août. Sur ce même site, en 2019 , les mâles sont les plus nombreux six jours avant le pic de vol des femelles.

\section{DÉPLACEMENTS}

Au cours des trois années de suivis, 959 déplacements de 431 individus ont été observés. La distance moyenne des déplacements est corrélée au nombre de recapture (KruskallWallis $\mathrm{H}=17,65 ; \mathrm{p}<0,01)$. Toutefois, la distance moyenne de déplacement ne présente pas de différences significatives entre les individus capturés trois et quatre fois (Mann-Whitney $\mathrm{U}=527 ; \mathrm{p}=0,41)$. Elle est de $86,46 \pm 6,34 \mathrm{~m}$ après au moins trois recaptures $(n=81)$.

La distance parcourue par les individus capturés au moins trois fois n'est pas différente entre les sites (Mann-Whitney $U=279 ; p=0,32$ ) et entre les sexes (Mann-Whitney $\mathrm{U}=254 ; \mathrm{p}=0,54)$.

La distance maximale observée entre deux captures est de $333 \mathrm{~m}$ sur le site de Moreuil, qui présente une longueur totale de $750 \mathrm{~m}$.

Sur la base des individus capturés trois fois $(n=66)$, quatre fois $(n=14)$ et cinq fois $(n=1)$, la méthode du MCP nous a permis de calculer les surfaces minimales fréquentées par 81 individus. Elles varient de 7 à 3769 m² $^{2}$ Entre 2013 et 2018 , la surface médiane occupée par les individus s'est réduite sur le site de Moreuil (Mann Whitney $U=27$; $p<0.05$ ). Évaluée à $2188 \mathrm{~m}^{2}$ en 2013, elle était estimée à $749 \mathrm{~m}^{2}$ en 2018 . 
A

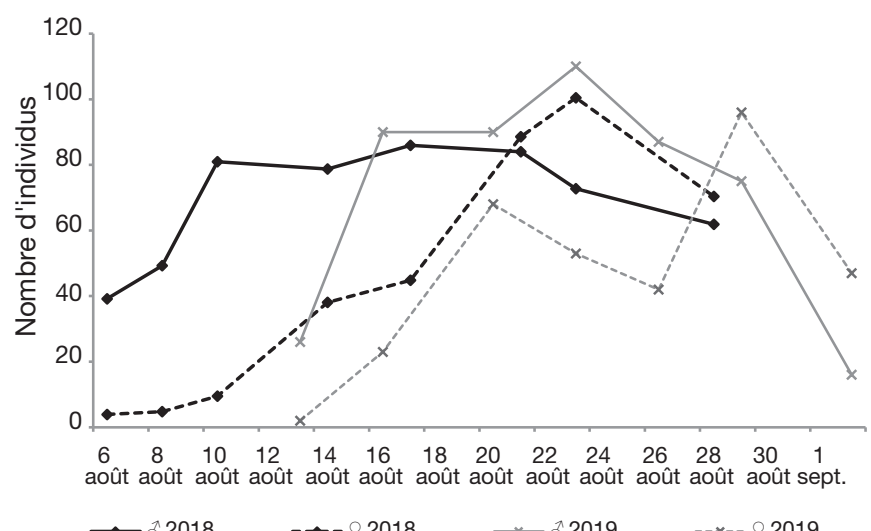

B

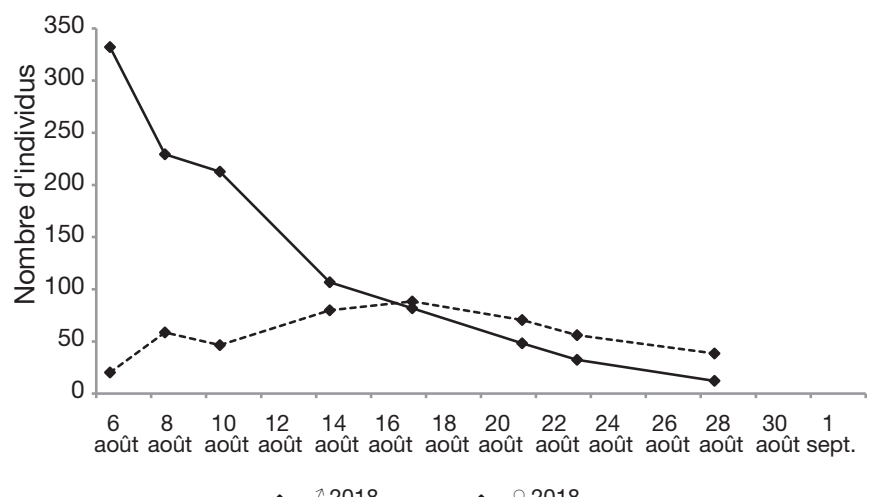

FIG. 6. - Taille de la population journalière des mâles et des femelles d'Arethusana arethusa (Denis \& Schiffermüller, 1775) sur les sites de Fignières en 2018 et 2019 (A) et de Moreuil (B) en 2018. Les estimations sont issues des données de capture-marquage-recapture analysées par le modèle POPAN dans le programme MARK. Les modèles utilisés sont présentés dans le Tableau 3.

À Fignières, l'aire d'occupation médiane n'est pas différente entre les années (Kruskall-Wallis $\mathrm{H}=0,12 ; \mathrm{p}=0,93$ ). Elle est estimée à $451 \mathrm{~m}^{2}$.

\section{UTILISATION DES MILIEUX PAR LES IMAGOS}

Les ratios de sélection appliqués aux végétations herbacées font ressortir qu'en période imaginale, $A$. arethusa sélectionne de manière préférentielle les pelouses pionnières à matures (RSPJM = 2,65). En revanche, les formations herbacées les moins diversifiées et les plus denses sont évitées sur les deux sites (RSOMS = 0,14) (Fig. 7).

Au cours des cinq sessions de suivis en 2013 et des six sessions en 2018, 627 observations de comportements ont été réalisées. $37 \%$ concernent des comportements de vol (principalement court), $32 \%$ de repos, $27 \%$ de butinage et $4 \%$ d'accouplement. $A$. arethusa a été observé en butinage sur 11 espèces de plantes à fleurs $(\mathrm{n}=169)$ (Fig. 8). Il s'agit d'espèces herbacées à floraison tardive, souvent caractéristiques des pelouses et prairies sèches et toutes considérées comme melliferes (Lambinon et al. 2004). Eryngium campestre L. et Scabiosa columbaria L. ressortent comme les deux plantes les plus utilisées sur les sites étudiés.

\section{HABITAT LARVAIRE}

Au total, 33 chenilles d'A. arethusa ont été trouvées sur 28 points différents. Elles ont été observées entre 23 h10 et 02 h30. Nous n'avons plus observé d'activité ensuite. $88 \%$ d'entre-elles ont été observées sur Festuca ovina Gr. Des chenilles ont également été trouvées sur Brachypodium pinnatum (L.) P.Beauv. et Hippocrepis comosa L.

L'ensemble des chenilles a été observé au sein de pelouses juvéniles à matures dépourvues d'arbustes. La comparaison des quadrats occupés et inoccupés par les chenilles révèle plusieurs différences significatives (Tableau 5). Les micro-habitats occupés $\operatorname{par} A$. arethusa se caractérisent par une hauteur de végétation rase $(6,57 \pm 2,39 \mathrm{~cm})$, un recouvrement plus important en fétuque $\left(22,6 \pm 9,54 \%\right.$ par $\left.\mathrm{m}^{2}\right)$ et de sol à nu $\left(8,21 \pm 7,35 \%\right.$ par $\left.\mathrm{m}^{2}\right)$. Les touffes de fétuque utilisées étaient situées en bordure d'écorchures et figuraient parmi les plus robustes des stations de présence.

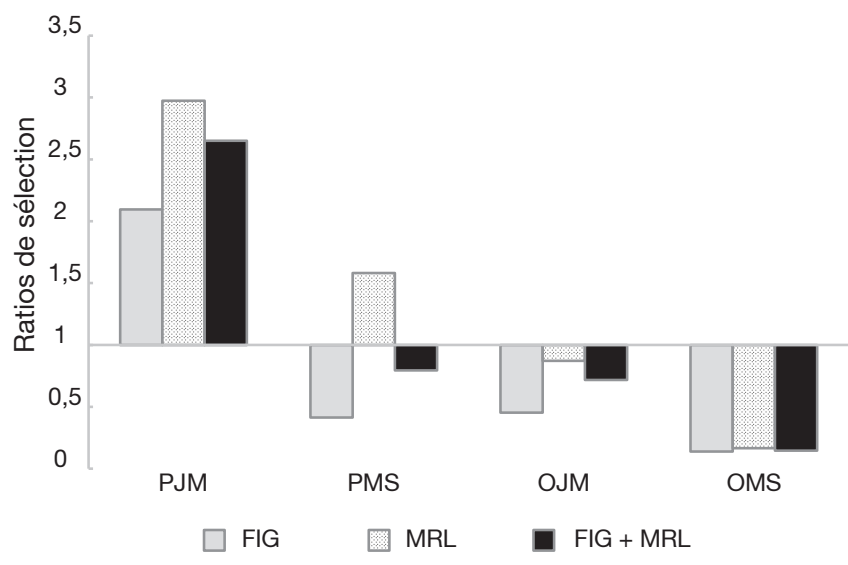

FIG. 7. - Ratios de sélection (Manly et al. 2002) des quatre habitats herbacés (PJM, PMS, OJM, OMS) sur les sites de Fignières (FIG) et Moreuil (MRL). Ratios de sélection calculés sur la base de la localisation des 2410 contacts d'Arethusana arethusa (Denis \& Schiffermüller, 1775) relevés en 2013, 2018 et 2019 ( $F I G=961$ contacts; $M R L=1449$ contacts). Un ratio supérieur à 1 indique une sélection positive et un ratio inférieur à 1 une sélection négative. Abréviations: OJM, ourlet juvénile à mature; OMS, ourlet mature à sénescent; PJM, pelouse juvénile à mature; PMS, pelouse mature à sénescente. Voir Tableau 1 pour la description des habitats.

\section{DISCUSSION ET APPLICATION POUR LA CONSERVATION}

\section{STRUCTURE DÉMOGRAPHIQUE DES POPULATIONS}

La méthode de capture-marquage-recapture a montré son efficacité puisque des estimations précises des tailles de populations ont pu être établies. Les marges d'erreur des estimations pour un niveau de confiance de $95 \%$ étaient comprises entre 13 et $19 \%$ selon les sites et les années. Entre 2013 et 2018, nous avons observé une importante baisse de la population totale d' $A$. arethusa et une certaine synchronie entre les deux populations étudiées. Entre 2018 et 2019, la taille de la population sur le site de Fignières est restée stable.

La déclinaison du Suivi Temporel des Rhopalocères de France (Manil \& Henry 2007) et du suivi des milieux ouverts par 


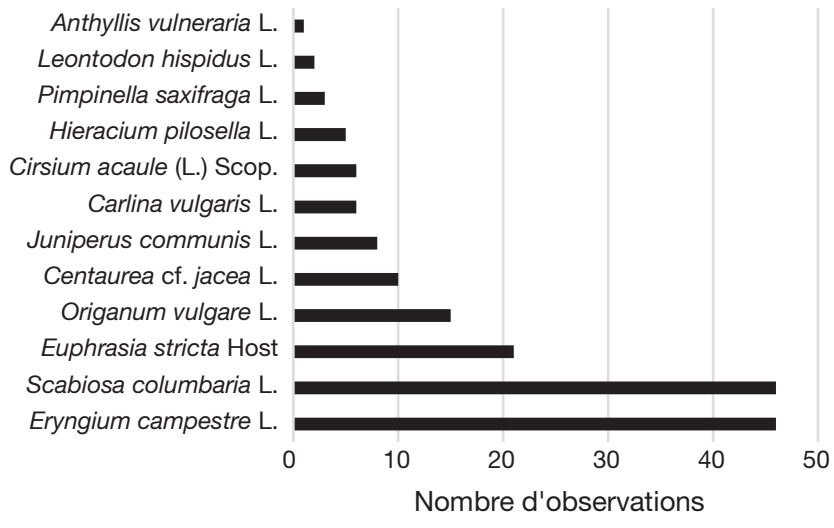

FIG. 8. - Observations de butinage d'Arethusana arethusa (Denis \& Schiffermüller, 1775) $(n=169)$ sur les trois points d'observations du site de Fignières (2015 et 2018).

les Rhopalocères dans les réserves naturelles de France (Langlois \& Gilg 2007) sur plusieurs sites du territoire national abritant des populations d' $A$. arethusa témoigne de résultats similaires: d'importantes variations interannuelles d'abondance sont observées et les abondances relevées en 2013 sur la plupart des stations de suivis étaient supérieures aux moyennes relevées sur la période 2010-2019 (Fig. 9).

Ces données permettent d'envisager que les différences d'effectifs observées entre 2013 et 2018 sur le site de Fignières ( $-54 \%)$, dont les actions conservatoires ont permis de maintenir les conditions stationnelles, reflètent ces variations interannuelles plutôt qu'un déclin de la population. Les prochains suivis permettront de le confirmer.

Une telle variation interannuelle de la taille de la population fait néanmoins peser un risque accru d'extinction sur les petites populations (Gabriel \& Bürger 1992; Bulman et al. 2007). Cela souligne l'importance de conserver des populations de plusieurs centaines d'individus pour réduire l'impact des processus stochastiques.

Sur le site de Moreuil, qui ne bénéficie pas d'actions conservatoires, la baisse d'effectifs observée entre 2013 et 2018 est plus accentuée $(-82 \%)$ que sur le site de Fignières (- $54 \%$ ). La baisse progressive de la taille de la population dès le mois d'août 2018 laisse suggérer des conditions stationnelles défavorables au développement des imagos cette année-là. Nous formulons l'hypothèse que la dynamique observée est liée à une réduction de la disponibilité en ressource faisant suite à une modification des pratiques d'entretien des ourlets calcicoles. En effet, contrairement au site de Fignières, $A$. arethusa se rencontre en abondance dans ces végétations (40\% des contacts contre $13 \%$ à Fignières). Considérant d'après nos résultats que les ourlets ne sont pas des habitats de ponte préférentiels, nous supposons qu'A. arethusa s'y déplace pour y trouver les ressources alimentaires limitées sur la pelouse. Or, depuis 2016, les ourlets calcicoles ne sont plus entretenus en automne mais en juillet, réduisant considérablement la disponibilité des ressources pour le papillon.

Si la disponibilité en ressource détermine la taille des populations (Pollard \& Yates 1992; Dennis 2012), elle est une composante qui influence, avec les conditions météorolo-
TABLEAU 5. - Moyennes ( \pm écartype) de la hauteur de la végétation, du recouvrement de la strate herbacé, de sol à nu et de fétuque relevées en 2018 au sein de quadrats de $1 \mathrm{~m}^{2}(n=208)$. Le test $U$ de Mann-Whithney est utilisé pour tester l'hypothèse selon laquelle la distribution des données est la même entre les quadrats occupés et non occupés par les chenilles d'Arethusana arethusa (Denis \& Schiffermüller, 1775). Abréviation: n.s., non significatif.

\begin{tabular}{lrrr}
\hline Paramètres & $\begin{array}{c}\text { Quadrat } \\
\text { occupé } \\
\mathbf{( N = 2 8 )}\end{array}$ & $\begin{array}{c}\text { Quadrat } \\
\text { non occupé } \\
\mathbf{( N = 1 8 0 )}\end{array}$ & $\begin{array}{c}\text { Test U } \\
\text { (Mann- } \\
\text { Whitney) }\end{array}$ \\
\hline $\begin{array}{l}\text { Hauteur moyenne de la } \\
\text { végétation }\end{array}$ & $6,57 \pm 2,39$ & $11,88 \pm 7,76$ & $\mathrm{p}<0,001$ \\
Recouvrement (\%) & & & \\
$\quad$ Strate herbacée & $94,28 \pm 9,09$ & $93,31 \pm 9,39$ & $\mathrm{n} . \mathrm{s}$. \\
$\quad \begin{array}{l}\text { Sol nu } \\
\text { Plante hote (fétuque) }\end{array}$ & $8,21 \pm 7,35$ & $6,29 \pm 9,59$ & $\mathrm{p}<0,05$ \\
& $22,6 \pm 9,54$ & $8,62 \pm 9,62$ & $\mathrm{p}<0,001$ \\
\hline
\end{tabular}

giques, la durée de vie des individus (Roy et al. 2001; Tudor et al. 2004; Beck \& Fiedler 2009). La persistance plus courte relevée sur le site de Moreuil (six jours en moyenne contre dix sur le site Fignières) renforce l'hypothèse d'une population contrainte par la disponibilité en ressource.

Évaluée de 22 à 30 jours en conditions contrôlés (Bink 1992), nos résultats soulignent que cette durée de vie peut être atteinte en milieu naturel. La durée de vie moyenne est toutefois bien plus courte, comprise entre six et douze jours selon les sites et les années. Au regard du décalage phénologique observé entre le pic de vol des mâles, et celui plus tardif et plus bref des femelles, les conditions météorologiques apparaissent également comme une composante essentielle du succès de reproduction de l'espèce. Cela suppose qu'en contexte océanique, les populations d' $A$. arethusa sont davantage affectées par les processus stochastiques qui en résultent, d'autant plus si les populations sont de petites tailles et isolées.

\section{DiSTRIBUTION SPATIALE, MOBILITÉ}

ET UTILISATION DES RESSOURCES

Dans un contexte de mise en œuvre de la politique dite "Trame verte et bleue» et parce que le fonctionnement métapopulationnel doit être recherché pour assurer une conservation durable de la plupart des papillons de jour (Thomas \& Jones 1993; Hanski \& Thomas 1994; Thomas 1995 ; Schtickzelle et al. 2005), la connaissance des capacités de déplacements d' $A$. arethusa est importante pour établir les stratégies de conservation. Dans la littérature, la faible mobilité d'A. arethusa est mentionnée mais n'est pas quantifiée (Pintureau 1976a, 1976b, 1977; Bink 1992; Reinhardt et al. 2007). Sur la base d'un modèle théorique lié aux traits de vie de l'espèce, Stevens et al. (2013) avaient estimé qu'A. arethusa possèdait une capacité de dispersion moyenne de l'ordre de $500 \mathrm{~m}$. Nos résultats ont permis de relever une distance de déplacement intra-site moyenne de 86,46 \pm 6,34 m et une distance maximale entre deux captures de $333 \mathrm{~m}$. Ces valeurs doivent être considérées comme des minima puisqu'elles sont fortement liées au contexte des sites étudiés qui sont de faibles superficies ( $<5 \mathrm{ha})$ et isolés comme en témoigne l'absence d'observation sur les sites potentiels périphériques. 


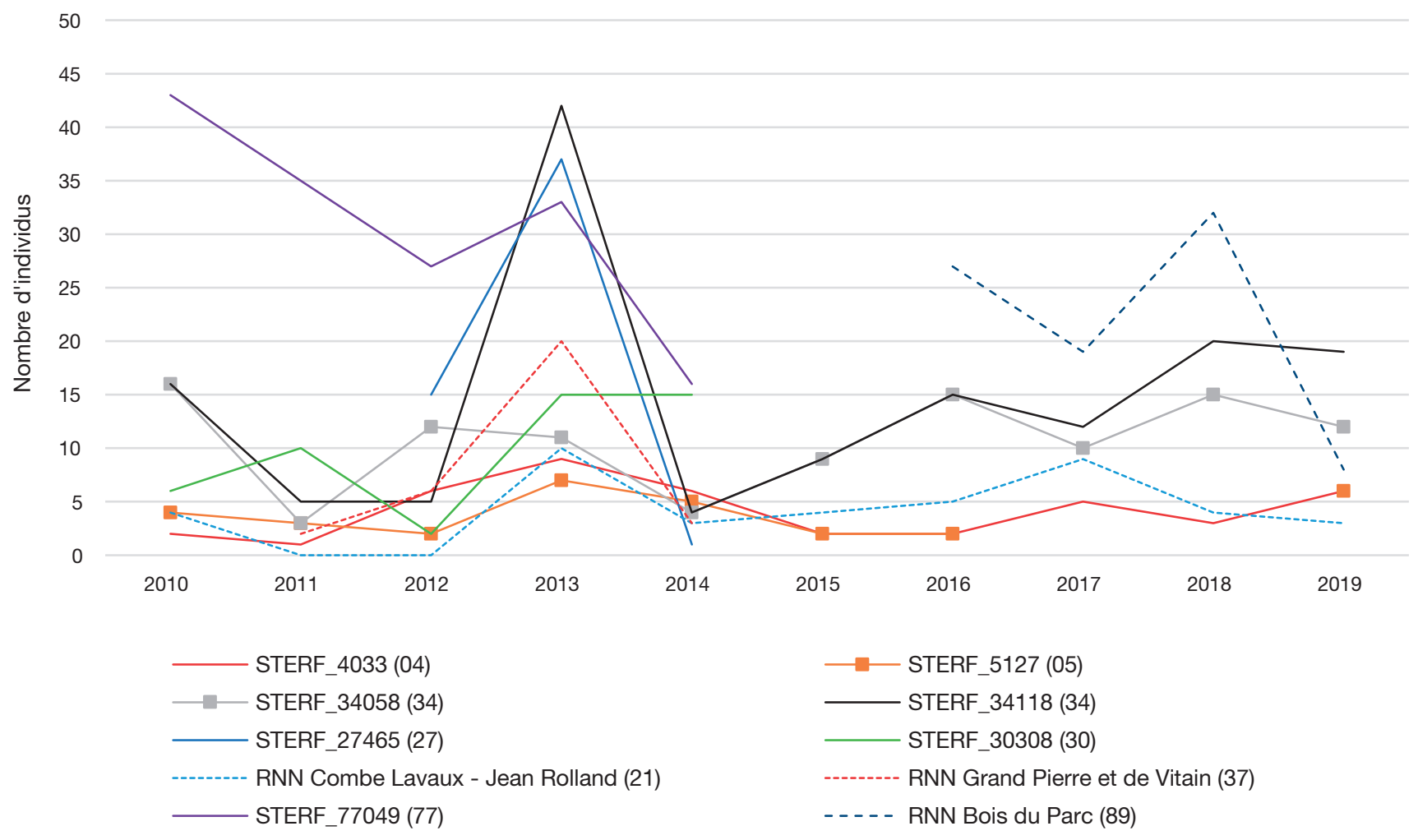

FIG. 9. - Nombre d'individus d'Arethusana arethusa (Denis \& Schiffermüller, 1775) observés sur des stations appliquant le Suivi temporel des Rhopalocères de France (Manil \& Henry 2007) (ligne continue) et suivi des milieux ouverts par les Rhopalocères dans les réserves naturelles de France (Langlois \& Gilg 2007) (pointillé). Les départements de rattachement sont donnés entre parenthèse. Abréviations: RNN, Réserves naturelles nationales; STERF, Suivi temporel des Rhopaloècres de France.

Des observations de terrain permettent néanmoins d'envisager qu' $A$. arethusa serait capable de parcourir plusieurs kilomètres. Ainsi, dans le département de l'Aisne, quatre individus ont été observés en 2015 sur un site de deux hectares géré et suivi depuis 2007 par le CEN Hauts-de-France (Gerard com. pers.). L'espèce n’a depuis plus été revue. Si les individus peuvent provenir d'une population non connue, l'hypothèse d'une colonisation depuis le camp militaire de Sissonne, situé à trois kilomètres et abritant la plus importante population régionale est envisagée. En région Centre-Val de Loire, $A$. arethusa est observé en petit effectif (< cinq individus) sur des pelouses situées entre un à deux kilomètres d'un site abritant une population plus importante (> 100 individus) (Lévêque 2013).

La notion de "surface minimale requise» est aussi une composante importante des stratégies de conservation car elle conditionne la persistance des populations (Baguette \& Stevens 2013). À l'échelle des individus, nous observons qu'A. arethusa peut accomplir son cycle imaginal sur des espaces restreints, de moins de $1000 \mathrm{~m}^{2}$. Ces résultats sont concordants avec des observations faites en région Centre-Val de Loire où des individus se rencontrent sur des pelouses rases de seulement $500 \mathrm{~m}^{2}$ (Gressette, com. pers.).

Nos résultats soulignent qu'une surface de deux hectares, correspondant à l'aire d'occupation d'A. arethusa sur le site de Fignières, peut accueillir une population de plus de 700 individus. Au regard des variations interannuelles d'effectifs et du maintien de cette population depuis 20 ans, nous considérons qu'une telle surface est nécessaire, au sein du domaine atlantique, pour assurer un maintien des populations sur le moyen terme.

Sur les sites étudiés, les pelouses et notamment les phases les plus pionnières apparaissent importantes dans le cycle de vie d'A. arethusa car elles abritent les habitats larvaires de l'espèce qui se caractérisent par une végétation herbacée rase avec une large proportion de sol à nu où se développent des touffes vigoureuses de Fétuque, sa plante hôte principale. Nous avons également observé qu'A. arethusa est souvent posé au sol au sein des pelouses rases. Ce comportement, que nous avons qualifié de repos, pourrait correspondre à une activité de thermorégulation décrite chez plusieurs Satyrinae (Hipparchia semele (Linnaeus, 1758) [Dreisig 1995], Hipparchia fagi (Scopoli, 1763) [Möllenbeck et al. 2009]) qui permettrait, selon Dreisig (1995), d'augmenter l'efficacité des vols.

Si les pelouses rases abritent une richesse floristique plus importante, en période de vol d'A. arethusa, ce sont les pelouses les plus denses et les ourlets, qui disposent de réserves hydriques plus importantes, qui offrent le plus de ressources florales nectarifères pour l'alimentation des adultes. Les rares plantes butinées par $A$. arethusa y sont associées: Eryngium campestre, Scabiosa columbaria, Origanum vulgare L., Centaurea jacea L.

\section{CONSERVATION}

Par sa faible répartition et son déclin manifeste, Arethusana arethusa fait partie de la liste des espèces menacées d'extinction en région Hauts-de-France (Picardie Nature 2016). La fragi- 
lité des populations régionales se confirme d'autant plus que l'on tient compte des exigences écologiques de l'espèce et des contextes des sites. $A$. arethusa présente de nombreux traits communs avec des espèces disparues des Hauts-de-France (Chazara briseis (Linnaeus, 1764) et Hipparchia statilinus (Hufnagel, 1766)) ou dont l'aire d'occupation s'est fortement réduite (Hipparchia semele n'étant plus présent que sur le littoral). Il apparaît aujourd'hui nécessaire de poursuivre et de renforcer les mesures de conservation pour éviter le risque stochastique d'extinction et permettre aux espèces de perdurer sur des milieux leur demeurant favorables. Ainsi, $A$. arethusa devra être intégré à la liste des espèces de "priorité régionale» dans le cadre la future déclinaison régionale du Plan national d'actions 2018-2028 en faveur des papillons de jour (Houard \& Jaulin 2018).

La perte d'habitat (embroussaillement, plantation de pins) constitue la principale cause de régression des populations d'A. arethusa en région Hauts-de-France. Depuis 30 ans, le CEN Hauts-de-France a entrepris de nombreuses démarches pour tenter de préserver les stations connues mais, faute de volontés locales, la plupart d'entre elles sont restées vaines et la disparition des populations n'a pu être que constatée.

À l'image du camp militaire de Sissonne (Aisne) où prospère sur près de 5000 hectares de pelouses et prairies sèches l'essentiel des effectifs régionaux d'A. arethusa, la conservation durable des populations se développant sur des espaces de petites tailles et fragmentés ne peut s'envisager sans la restauration d'un fonctionnement métapopulationnel dans un réseau fonctionnel de sites (Thomas \& Jones 1993; Hanski \& Thomas 1994; Thomas 1995; Schtickzelle et al. 2005).

Si cet objectif doit être recherché, la conservation à court terme des populations est une problématique à laquelle se confronte de nombreux gestionnaires d'espaces naturels qui doivent faire coexister, sur des espaces souvent de faibles superficies, des dynamiques spatiales et temporelles de la végétation permettant d'assurer une disponibilité en ressources et en habitats nécessaires à l'accomplissement du cycle de vie des espèces. L'importance du pâturage pour la conservation de Satyrinae associés aux pelouses steppiques est bien documentée (Wallis De Vries et al. 1998; Kadlec et al. 2009; Bubová et al. 2015 ; Lafranchis 2001 ; Lafranchis et al. 2015). En phase d'entretien, le pâturage tardif (septembre/octobre) conduit avec une certaine intensité, sur une courte période, paraît être le meilleur compromis pour bloquer la dynamique de la végétation tout en préservant les ressources nectarifères en période de vol. Parce que cette gestion peut impacter les premiers stades larvaires qui se nourrissent de jour, elle doit s'inscrire dans un système rotatif avec la conservation d'une large proportion de zones refuges non pâturées. Le pâturage hivernal peut être une alternative intéressante sur des pelouses en bon état de conservation mais doit être complété périodiquement par une action en période de développement de la végétation pour limiter le développement des graminées sociales.

Lorsque les pressions exercées par la faune sauvage (lapins, chevreuils, sangliers) sont suffisantes pour garantir le maintien d'habitats pionniers, la fauche exportatrice peut constituer une alternative moins contraignante pour garantir une structure verticale basse et diversifiée. Sur les pelouses enfrichées, elle permet de réduire considérablement le recouvrement des espèces sociales (Brachypodium pinnatum, bromes) et de reconstituer assez rapidement (trois à cinq ans) des pelouses très fleuries (Delescaille 2006). Si en phase de restauration le fauchage en période de végétation est le plus efficace pour reconstituer un tapis végétal fleuri, la fauche réalisée en fin d'été (septembre/octobre) et par rotation permet de réduire les impacts sur l'entomofaune.

Quelles que soient les modalités de gestion mise en œuvre, il convient de maintenir l'ensemble des stades de la série de végétation des pelouses sèches, depuis les pelouses pionnières jusqu'aux fourrés arbustifs. Les pelouses fermées et jeunes ourlets offrent des ressources pour le papillon qui peuvent être limitées sur les pelouses rases et écorchées au mois d'août. Également, les fourrés arbustifs constituent des refuges pour $A$. arethusa la nuit ou lorsque les conditions météorologiques sont mauvaises (Obs. pers.).

Nos résultats soulignent les limites des actions de conservation réalisées à l'échelle de stations ou parcelles qui n'affranchissent pas les populations des risques stochastiques. Cette diversité fonctionnelle d'habitats doit également être déclinée à l'échelle des territoires pour favoriser les déplacements des individus. Parce que l'espèce semble avoir une capacité de dispersion limitée, nous considérons qu'une distance de deux kilomètres environ est à rechercher entre les sites pour maintenir des échanges d'individus. Si les habitats favorables abritant les noyaux populationnels (stations) doivent présenter une surface minimale de deux à trois hectares, les sites relais pourraient être de faible superficie, de l'ordre de plusieurs centaines de $\mathrm{m}^{2}$. Dans les territoires dominés par les grandes cultures, les prairies permanentes ou temporaires ainsi que les jachères et bandes enherbées présentent un rôle fonctionnel de premier ordre en tant que corridors de dispersion entre les pelouses calcicoles mais aussi comme habitat de substitution. Ces continuums herbacés doivent donc être pérennisés, renforcés, et tenir compte des exigences écologiques des espèces les plus menacées dans leurs modalités d'entretien (fauche tardives, bandes refuges, rotation, semis d'espèces végétales locales, etc.) (Fig. 10). L'atteinte de cet objectif nécessite d'impliquer les usagers locaux (exploitants agricoles, exploitants forestiers, gestionnaires de bermes routières, etc.) en s'appuyant sur des dispositifs de développement des territoires tels que les mesures agri-environnementales (MAE) et les contrats Natura 2000. Les mesures du type des MAE «Corridors pelouses» développées un temps par la Chambre d'Agriculture de Picardie vont dans ce sens et méritent d'être poursuivies et étendues. Cet objectif de renforcer la conservation des espèces prioritaires au sein des trames agricoles et forestières est traduit en action prioritaire dans le Plan d'action national en faveur des papillons de jour (Houard \& Jaulin 2018). Des actions concrètes sont ainsi attendues dans le cadre de sa déclinaison "Hauts-de-France» pour espérer conserver durablement les populations de papillons de jour inféodées aux pelouses calcicoles. 


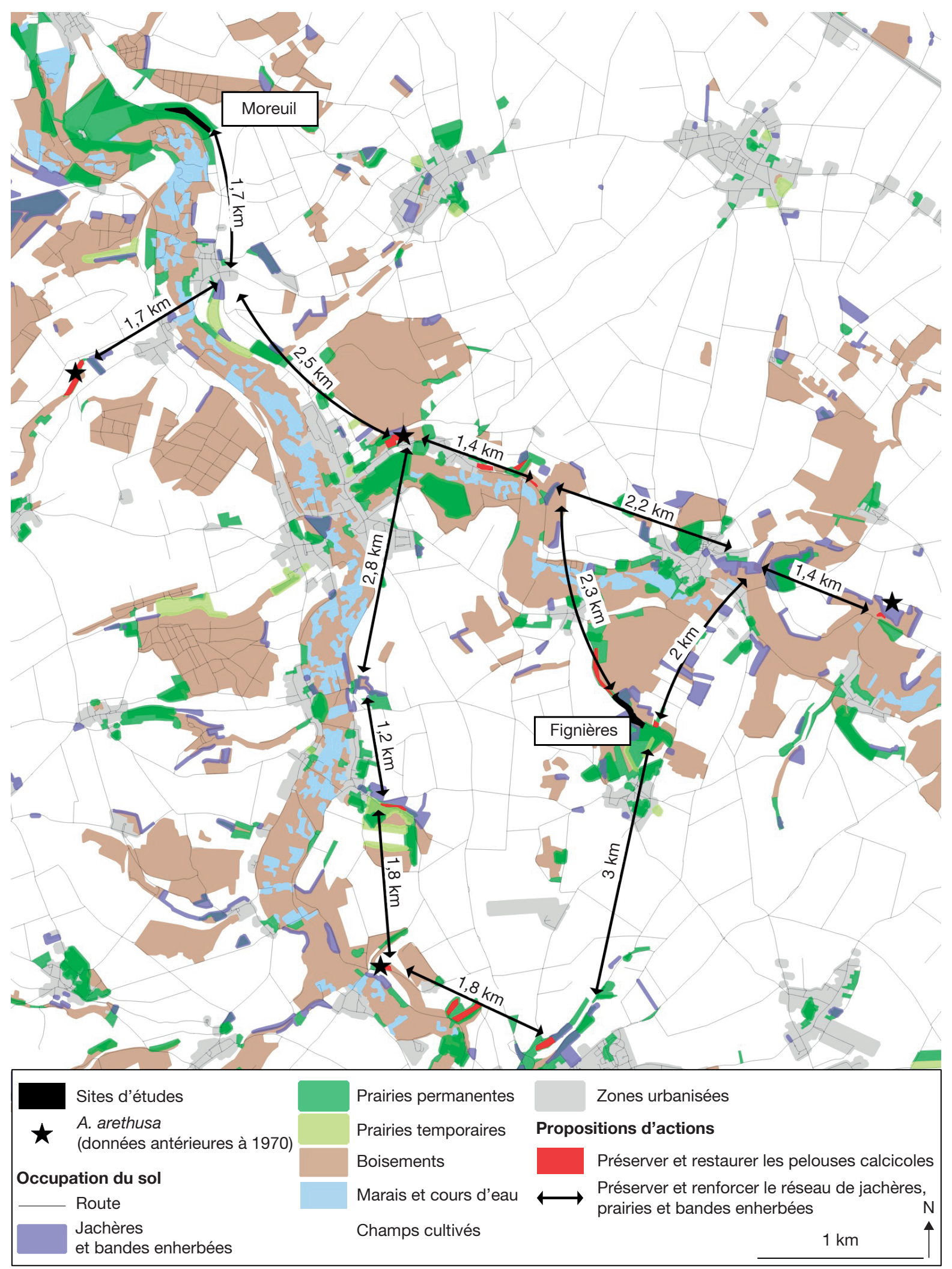

FIG. 10. - Localisation des sites de Moreuil et Fignières dans la matrice paysagère et proposition d'actions pour favoriser les déplacements d'Arethusana arethusa (Denis \& Schiffermüller, 1775) au sein du réseau de sites. Fond de carte: RPG 2019 @ IGN; OCS 2010 @) IGN - Paris. 


\section{Remerciements}

Ce travail n'aurait pu être accompli sans la participation aux campagnes de terrain de plusieurs bénévoles et stagiaires du CEN Hauts-de-France. Merci à Picardie Nature pour la mise à disposition des données régionales, au Muséum national d'Histoire naturelle pour la transmission des données du Suivi TEmporel des Rhopalocères de France et aux gestionnaires du réseau des conservatoires d'espaces naturels pour les échanges d'informations.

Nous remercions Xavier Houard et Aurélien Besnard pour leur relecture et propositions d'amélioration.

Les activités de connaissances et de conservation des espaces naturels dont découle ce travail sont soutenues financièrement par les partenaires institutionnels du CEN Hauts-de-France: Union Européenne, État, Région Hauts-de-France, Conseils départementaux de la Somme, de l'Oise et de l'Aisne ainsi que les Agences de l'Eau Artois-Picardie et Seine-Normandie.

\section{RÉFÉRENCES}

BACHelard F. \& Fournier F. (coord.) 2013. - Liste rouge des espèces menacées en Auvergne - Rhopalocères et zygènes. Société d'Histoire naturelle Alcide-d'Orbigny, Association entomologique d'Auvergne, DREAL Auvergne, Clermont-Ferrand, Aubière, 9 p.

Baguette M. \& Stevens V. M. 2013. - Predicting minimum area requirements of butterflies using life-history traits. Journal of Insect Conservation 14 (4): 645-652. https://doi.org/10.1007/ s10841-013-9548-x

BAillet Y. \& Guicherd G. 2018. - Dossier de présentation de la liste rouge Rhopalocères \& Zygènes de Rhône-Alpes. Flavia APE, Trept, 19 p.

BECK J. \& FIEDLER K. 2009. - Adult life spans of butterflies (Lepidoptera: Papilionoidea + Hesperioidea): broadscale contingencies with adult and larval traits in multi-species comparisons. Biological Journal of the Linnean Society 96 (1): 166-184. https://doi. org/10.1111/j.1095-8312.2008.01102.x

BENCE S. (coord.), 2014. — Liste rouge régionale des Rhopalocères et Zygènes de Provence-Alpes-Côte d'Azur. CEN PACA, Aix-enProvence, $22 \mathrm{p}$.

BINK F. A. 1992. - Ecologische Atlas van de Dagulinders van Noordwest-Europa. Schuyt \& Co., Bloemendaal, 512 p.

BOUlET V. 1986. - Les pelouses calcicoles (Festuco-Brometea) du domaine atlantique français et ses abords au nord de la Gironde et du Lot: essai de synthèse phytosociologique. Rapport de thèse, Université de Lille, 333 p.

Bubova T., Vrabec V., Kulma M. \& Nowicki P. 2015. — Land management impacts on European butterflies of conservation concern: a review. Journal of Insect Conservation 19 (5): 805-821. https://doi.org/10.1007/s10841-015-9819-9

Buckland S. T., Anderson D. R., Burnham K. P., LaAKe J. L., BorChers D. L. \& ThOMAs L. 2001. — Introduction to Distance Sampling. Oxford University Press, 448 p.

Bulman C. R., Wilson R. J., Holt A. R., Galvez-Bravo L., Earky R. I., Warren M. S. \& Thomas C. D. 2007. - Minimum viable metapopulation size, extinction debt, and the conservation of a declining species. Ecological Applications 17 (5): 1460-1473. https://doi.org/10.1890/06-1032.1

BURNHAM K. P. \& ANDERSON D. R. 2002. - Model Selection and Inference: a Practical Information-Theoretic Approach - 2nd Edition. Springer-Verlag, New York, 488 p.

Coppa G., Grange P., Lambert J.-L., Leconte R., Sauvage A., TERNOIS V. 2007. — Liste rouge des insectes de Champagne-Ardenne. DIREN Champagne-Ardenne, Châlons-en-Champagne, 6 p.

DeLESCAILLE L.-M. 2006. — La restauration des pelouses calcicoles en Région wallonne: aspects scientifiques et techniques (première partie). Parcs et Réserves 61 (4): 4-11.

DENNIS R. L. H. 2012. - A Resource-Based Habitat View for Conservation: Butterflies in the British Landscape. Wiley-Blackwell, Chichester, 420 p. https://doi.org/10.1002/9781444315257

DEWULF L. \& HOUARD X. (coord.) 2016. - Liste rouge régionale des Rhopalocères et des Zygènes d'Île-de-France. ARB îdF, Office pour les Insectes et leur Environnement, Association des Lépidoptéristes de France, Paris, 88 p.

DreISIG H. 1995. - Thermoregulation and flight activity in territorial male graylings, Hipparchia semele (Satyridae), and large skippers, Ochlodes venata (Hesperiidae). Oecologia 101 (2): 169176. https://doi.org/10.1007/BF00317280

DutolT T. 1996. - Dynamique et gestion des pelouses calcaires de Haute-Normandie - Nouvelle édition. Presses universitaires de Rouen et du Havre, Mont-Saint-Aignan, 220 p. https://doi. org/10.4000/books.purh.8075

Ebert G., Hofmann A., Karbiener O., MeineKe J.-U., Steiner A. \& TRusCH R. 2008. - Rote Liste und Artenverzeichnis der Großschmetterlinge Baden-Württembergs (Stand: 2004). LUBW Landesanstalt für Umwelt, Messungen und Naturschutz Baden-Württemberg, Karlsruhe, 64 p.

FARTMANN T. \& HERMAnN G. 2006. — Larvalökologie von Tagfaltern und Widderchen in Mitteleuropa - von den Anfängen bis heute, in Fartmann T. \& G. HermanN G. (éds), Larvalökologie von Tagfaltern und Widderchen in Mitteleuropa. Abhandlungen aus dem Westfälischen Museum für Naturkunde 68 (3-4): 11-57.

FranÇOIS P. 2003. — Les Rhopalocères du Nord-Pas-de-Calais (observations 2000-2002). Cercle des Lépidoptéristes de Belgique $32(2-4)$ : 46-73.

GABRIEL W. \& BÜRGER R. 1992. - Survival of small populations under demographic stochasticity. Theoretical Population Biology 41 (1): 44-71. https://doi.org/10.1016/0040-5809(92)90049-Y

GEIGER W. (coord.) 1987. - Les Papillons de jour et leurs biotopes: espèces, dangers qui les menacent, protection. Ligue suisse pour la Protection de la Nature, Egg, 512 p.

GouvernanCe RÉGIONALE DE LA Biodiversité Hauts-De-FranCe (GRéB Hauts-DE-FranCE) 2020. — Guide Nature en Hautsde-France - Édition 2020. Observatoire de la biodiversité des Hauts-de-France, Bailleul, 192 p.

Haddad N. M., Hudgens B., Damiani C., Gross K., Kuefler D. \& POLLOCK K. 2008. — Determining optimal population monitoring for rare butterflies. Conservation Biology 22 (4): 929-940. https://doi.org/10.1111/j.1523-1739.2008.00932.x

HANSKI I. \& ThOMAS C. D. 1994. - Metapopulation dynamics and conservation: a spatially explicit model applied to butterflies. Biological Conservation 68 (2): 167-180. https://doi. org/10.1016/0006-3207(94)90348-4

HOUARD X. \& JAULIN S. (coord.) 2018. — Plan national d'actions en faveur des "Papillons de jour»-Agir pour la préservation de nos lépidoptères diurnes patrimoniaux 2018-2028. Office pour les Insectes et leur Environnement, DREAL Auvergne-Rhône-Alpes, Ministère de la Transition écologique et solidaire, La Défense, 64 p. https://doi.org/10.13140/RG.2.2.34233.21602

IMAGO 2014. - La Liste rouge des Rhopalocères et Zygènes menacés en Alsace. IMAGO, ODONAT, Strasbourg, 25 p.

Jugan D., Mora F., Robert J.-C., Prot J.-M., Rousset S. \& BetTINELLI L. 2013. - Listes rouges régionales d'insectes de FrancheComté. Libellules (Odonates), Criquets, Sauterelles et Grillons (Orthoptères), Papillons de jour (Rhopalocères \& Zygènes) et Mantes (Mantidés). OPIE, Conservatoire botanique national de FrancheComté, Observatoire régional des Invertébrés, Besançon, 12 p.

Kadlec T., Vrba P. \& Konvicka M. 2009. - Microhabitat requirements of the critically endangered butterfly Chazara briseis (L.) (Nymphalidae, Satyrinae) in the Czech Republic. Nota lepidopterologica 32: 39-46.

LAFRANCHIS T. 2001. - Écologie et biologie de l'Hermite (Chazara briseis Linné, 1758) sur les causses du Quercy (Lot, France) 
(Lepidoptera: Nymphalidae, Satyrinae). Linneana Belgica 18 (2): 65-72.

LAFRANCHis T., Jutzeler F., Guillosson J.-Y. \& KAN B. 2015. La Vie des Papillons - Écologie, biologie et comportement des Rhopalocères de France. Diatheo, Esserts-Blay, 752 p.

Lambinon J., Delvosalle L. \& Duvigneaud J. 2004. - Nouvelle Flore de la Belgique, du Grand-Duché du Luxembourg, du Nord de la France et des Régions voisines. Cinquième édition. Édition du Patrimoine du Jardin botanique national de Belgique, Meise, $1167 \mathrm{p}$.

LANGLOIS D. \& GILG O. 2007. - Méthode de suivi des milieux ouverts par les Rhopalocères dans les Réserves naturelles de France. Réserves naturelles de France, Quétigny, 30 p.

Lett J.-M., Cama A., Faucheux F., Gressette S., Lévêque A. \& VANDROMME D. 2007. — Liste rouge des Lépidoptères de la région Centre. Nature Centre, Orléans, 8 p.

LÉVÊQUe A. 2013. - Étude entomologique. Trame Verte et Bleue de la région Centre. Lépidoptères. Fournitures d'éléments sur les Lépidoptères pour la détermination des réservoirs de biodiversité et des corridors associés. Entomo Fauna, DREAL Centre, Beaugency, 36 p.

LORTHIOIS M. (coord.) 2015. — Liste Rouge des Papillons diurnes ef Zygènes de Haute-Normandie. Conservatoire d'Espaces naturels de Haute-Normandie, Rouen, Saint-Étienne-du-Rouvray, 17 p.

louboutin B., Jaulin S., Charlot B. \& Danflous S. (coord.) 2019. - Liste rouge des Lépidoptères Rhopalocères et Zygènes d'Occitanie. Rapport d'évaluation. OPIE, CEN MP \& CENLR, Montferrier, Lez, $304 \mathrm{p}$.

Manly B. F. J., McDonald L. L., Thomas D. L., McDonALD T. L. \& ERICKSON W. P. 2002. - Resource Selection by Animals Statistical Design and Analysis for Field. Springer, Dordrecht, 222 p. https://doi.org/10.1007/0-306-48151-0

Manil L. \& Henry P.-Y. 2007. - Suivi temporel des Rhopalocères de France (STERF) - Suivi temporel des Insectes communs (STIC) Protocole national-Mars 2007. Observatoire de biodiversité Vigie nature, Département d'Écologie du Muséum national d'Histoire naturelle, Paris, $10 \mathrm{p}$

Meire G. \& Riviere G. 2015. - Le Marais et le coteau de Genonville (Moreuil, Somme) - Plan de gestion 2017-2026. Conservatoire d'espaces naturels de Picardie, Dury, 67 p. + annexes.

Meire G. \& Riviere G. 2016. - La Montagne de Fignières - Plan de gestion 2017-2026. Conservatoire d'espaces naturels de Picardie, Dury, 54 p. + annexes.

Mollenbeck V., Hermann G. \& Fartman T. 2009. — Does prescribed burning mean a threat to the rare satyrine butterfly Hipparchia fagi? Larval-habitat preferences give the answer. Journal of Insect Conservation 13: 77-87. https://doi.org/10.1007/ s10841-007-9128-Z

Nowicki P., Witek M., Skorka P., SetTele J. \& Woyciechowski M. 2005. - Population ecology of the endangered butterflies Maculinea teleius and $M$. nausithous and the implications for conservation. Population Ecology 47 (3): 193-202. https://doi. org/10.1007/s10144-005-0222-3

OAFS (coord.) 2019. - La Liste rouge des Papillons de jour d'Aquitaine. Observatoire Aquitain de la Faune Sauvage, Talence, 16 p.

PICARDIE NATURE (coord.) 2016. — Listes rouges régionales de la faune menacée de Picardie. Les Chiroptères, les Mammiferes terrestres, les Mammiferes marins, les Amphibiens/Reptiles, les Araignées "orbiteles", les Coccinelles, les Orthoptères, les Odonates, les Rhopalocères et Zygènes. Picardie Nature, Amiens, 41 p.

Pintureau B. 1976a. - Contribution à l'étude du genre Arethusana De Lesse [Lep. Satyridae]. Première partie: Étude zoogéographique. Alexanor 9: 243-250.

Pintureau B. 1976b. - Contribution à l'étude du genre Arethusana De lesse [Lep. Satyridae] (suite). Alexanor 9: 317-322.

Pintureau B. 1977. - Contribution à l'étude du genre Arethusana H. De Lesse. Résumé des parties II et III et compléments [Lep. Satyridae] (suite et fin). Alexanor 10: 98-104.

Poitou-Charentes Nature 2019. - Liste rouge du Poitou-
Charentes: chapitre Rhopalocères. Service Éditions LPO, Fontainele-Comte, $16 \mathrm{p}$.

POLLARD E. 1977. - A method for assessing changes in the abundance of butterflies. Biological Conservation 12 (2): 115-134. https://doi.org/10.1016/0006-3207(77)90065-9

POllaRd E. \& YATES T. J. 1992. - The extinction and foundation of local butterfly populations in relation to population variability and other factors. Ecological Entomology 17 (3): 249-254. https:// doi.org/10.1111/j.1365-2311.1992.tb01055.x

QGIS DEVELOPMENT TEAM 2016. - Q GIS Geographic Information System. Open Source Geospatial Foundation Project. http://www. qgis.org, dernière consultation le 12 novembre 2020.

Reinhardt R., Sbieschne H., Settele J., Fischer U. \& FieDLER G. 2007. - Tagfalter von Sachsen, in KLAusnitzer B. \& ReINHARDT R. (éds), Beitraege zur Insektenfauna Sachsens Band 6. Entomologische Nachrichten und Berichte, Beiheft 11, Dresden: $696 \mathrm{p}$

REINHARDT R. \& Bolz R. 2011. - Rote Liste und Gesamtartenliste der Tagfalter (Rhopalocera) (Lepidoptera: Papilionoidea et Hesperioidea) Deutschlands. Naturschutz und Biologische Vielfalt 70 (3): 167-194

Roy D. B., Rothery P., Moss D., Pollard E. \& Thomas J. A. 2001. - Butterfly numbers and weather: predicting historical trends in abundance and the future effects of climate change. Journal of AnimalEcology 70 (2): 201-217. https://doi.org/10.1111/ j.1365-2656.2001.00480.x

RUFFONI A. (coord.) 2015. - Élaboration d'une Liste rouge des Rhopalocères et Zygènes de Bourgogne - Période 2003-2012. Société d'histoire naturelle d'Autun, Autun, $11 \mathrm{p}$.

Schtickzelle N., Choutt J., Goffart P., Fichefet V. \& Baguette M. 2005. - Metapopulation dynamics and conservation of the marsh fritillary butterfly: population viability analysis and management options for a critically endangered species in Western Europe. Biological Conservation 126 (4): 569581. https://doi.org/10.1016/j.biocon.2005.06.030

Settele J., Kudrna O., Harpke A., Kühn I., Van Swaay C., Verovinik R., Warren M., Wiemers M., Hanspach J., HickLer T., KÜHn E., Van Halder I., Veling K., Vliegenthaty A., WyNHOFF I. \& SCHXEIGER O. 2008. - Climatic risk atlas of European butterflies. BioRisk 1: 1-712. https://doi.org/10.3897/ biorisk. 1

Stevens V., Trochet A., Blanchet S., Moulherat S., Clobert J. \& Baguette M. 2013. - Dispersal syndromes and the use of life-histories to predict dispersal. Evolutionary Applications 6 (4): 630-642. https://doi.org/10.1111/eva.12049

THOMAS J. A. 1983. - A quick method for estimating butterfly numbers during surveys. Biological Conservation 27 (3): 195-211. https://doi.org/10.1016/0006-3207(83)90019-8

THOMAS J. A. 1991. - Rare species conservation: case studies of European butterflies, in Spellerberg I. F., GoldSMiTH F. B. \& MORRIS M. G. (éds), The Scientific Management of Temperate Communities for Conservation. 31 st symposium of the British Ecological Society, Blackwell, Oxford: 149-197.

THOMAS J. A. 1995. - Ecology and conservation of butterfly metapopulations in the fragmented British landscape, in PULLIN A. S. (éd.), Ecology and Conservation of Butterflies: 46-63. https://doi. org/10.1007/978-94-011-1282-6_4

Thomas C. D. \& Jones T. M. 1993. - Partial recovery of a Skipper Butterfly (Hesperia comma) from population refuges: lessons for conservation in a fragmented landscape. Journal of Animal Ecology 62 (3): 472-481. https://doi.org/10.2307/5196

Tudor O., Dennis R. L. H., Greatorex-Davies J. N. \& SPARKS T. H. 2004. - Flower preferences of woodland butterflies in the UK: nectaring specialists are species of conservation concern. Biological Conservation 119 (3): 397-403. https://doi. org/10.1016/j.biocon.2004.01.002

UICN FRANCE, MNHN, OPIE \& SEF 2014. - La Liste rouge des espèces menacées en France - Chapitre Papillons de jour de France 
métropolitaine. UICN France, MNHN, OPIE, SEF, Paris, 16 p. VAN SWAaY C. A. M. \& WARREN M. S. 1999. — Red Data book of European butterflies (Rhopalocera). Council of Europe Publishing (Nature and Environment, No. 99), Strasbourg, 203 p. + annexes.

Van SwaAy C., Warren M. \& Lois G. 2006. - Biotope use and trends of European butterflies. Journal of Insect Conservation 10: 189-209. https://doi.org/10.1007/s10841-006-6293-4

Van Swaay C., Cuttelod A., Collins S., Maes D., López Munguira M., Šašić M., SetTele J., Veronik R., Verstrael T., Warren M., Wiemers M. \& Wynhof I. 2010. — European Red List of Butterflies. Publications Office of the European Union, Luxembourg, 26 p. + annexes.

Wallis De Vries M. F., Bakker J. P. \& Van Wieren S. E (éds) 1998. - Grazing and Conservation Management. Springer Netherland, Dordrecht, 374 p. https://doi.org/10.1007/97894-011-4391-2

Warren M. S., Hill J. K., Thomas J. A., Asher J., Fox R., Huntley B., Roy D. B., Telfer S., JefFcoate P., Hardling P., JeffCoAte G., Willis S. G., GreatoreX-Davies J. N., Moss D. \&
ThOmas D. 2001. - Rapid responses of British butterflies to opposing forces of climate and habitat change. Nature 414: 65-69. https://doi.org/10.1038/35102054

Wermeille E., ChitTARo Y. \& GONSETH Y. 2014. — Liste rouge Papillons diurnes et Zygènes. Espèces menacées en Suisse, état 2012. L'environnement pratique 1403, Office fédéral de l'environnement, Berne, et Centre Suisse de Cartographie de la Faune, Neuchâtel, $97 \mathrm{p}$.

White G. C. \& GARROT R. A. 1990. - Analysis of wildlife radiotracking data. Academic press, New York, 383 p. https://doi. org/10.1016/B978-0-08-092657-5.50008-2

White G. C. \& Burnham K. P. 1999. — Program MARK: survival estimation from populations of marked animals. Bird Study 46: 120-139. https://doi.org/10.1080/00063659909477239

Zografou K., Kati V., Grill A., Wilson R. J., Tzirkalli E., Pamperis L. N. \& Halley J. M. 2014. — Signals of climate change in butterfly communities in a mediterranean protected area. PLoS ONE 9 (1): e87245. https://doi.org/10.1371/journal. pone. 0087245 